\title{
Partial-symmetry-breaking phase transitions
}

\author{
Masanori Hanada ${ }^{1}$ and Brandon Robinson $\circledast^{2,1}$ \\ ${ }^{1}$ STAG Research Centre, University of Southampton, Southampton SO17 1BJ, United Kingdom \\ ${ }^{2}$ Instituut voor Theoretische Fysica, KU Leuven, Celestijnenlaan 200D, B-3001 Leuven, Belgium
}

(Received 16 June 2020; accepted 13 October 2020; published 12 November 2020)

\begin{abstract}
We demonstrate a novel feature of certain phase transitions in theories with large rank symmetry group that exhibit specific types of all-to-all interactions, a typical example being the matrix model description of a large- $N$ gauge theory. Recently, it has been pointed out that nontrivial features of the confinement/ deconfinement transition are understood as consequences of the coexistence of the confined and deconfined phases on the group manifold describing the color degrees of freedom. While these novel features of the confinement/deconfinement transition are analogous to the two-phase coexistence at the first-order transition of more familiar local theories, various differences such as the partial breaking of the symmetry group appear due to the all-to-all interaction. In this article, we show that similar phase transitions with partially broken symmetry can exist in various examples from quantum field theory and string theory. Our examples include the deconfinement and chiral transition in QCD, Gross-Witten-Wadia transition in two-dimensional lattice gauge theory, Douglas-Kazakov transition in two-dimensional gauge theory on sphere, and black hole/black string transition. In some of these examples, we give new interpretations to previously known solutions and clarify the underlying physical mechanism governing their phase behavior.
\end{abstract}

DOI: 10.1103/PhysRevD.102.096013

\section{INTRODUCTION}

In this paper, we suggest a generic feature of the phase transitions in local quantum field theories with large rank symmetry groups and all-to-all couplings between internal degrees of freedom. By this, we mean that our analysis will focus primarily on theories where the interactions in spacetime are local but, e.g., the color or flavor degrees of freedom see all-to-all couplings. To further clarify the language that we will be using throughout the paper, we will refer to the background spacetime on which local physics takes place as physical space and the background on which the degrees of freedom experience all-to-all coupling as internal space. For example, consider a gauge theory with a field $\Phi_{i j}(x)$ in the adjoint representation, where $x$ is the coordinate of the physical space and $i, j=$ $1,2, \ldots, N$ are regarded as "coordinates" in the internal space. We can thus imagine that the internal space consists of $N^{2}$ points, and the degrees of freedom at each point interacts with all others; it is in this sense that the internal space interactions are nonlocal. While we almost exclusively study large- $N$ gauge theories in order to realize

Published by the American Physical Society under the terms of the Creative Commons Attribution 4.0 International license. Further distribution of this work must maintain attribution to the author(s) and the published article's title, journal citation, and DOI. Funded by SCOAP ${ }^{3}$. concretely the physics of the phase transitions of interest, the logic of the underlying mechanism appears to be more broadly applicable.

As a specific example, let us consider the confinement/ deconfinement transition in gauge theory [1-4] and consider the useful analogy with to the liquid-solid transition in water. A cartoon picture of the phase diagram of water at $1 \mathrm{~atm}$ and near $0{ }^{\circ} \mathrm{C}$ is shown in Fig. 1. Although the terminology of internal and physical space in the case of analyzing the liquid-solid transition of water is a bit
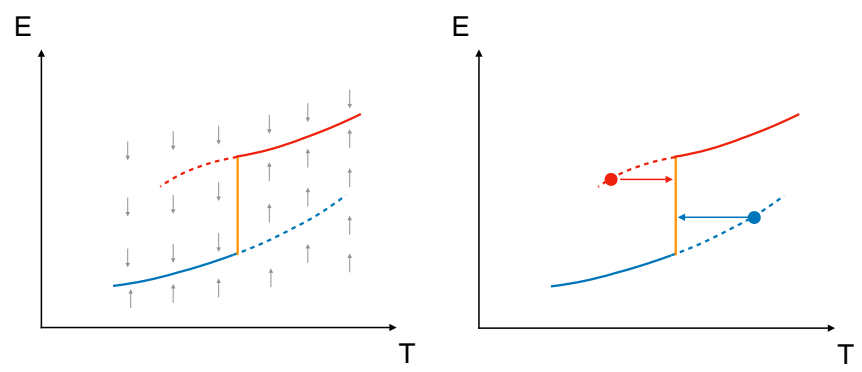

FIG. 1. A sketch of the phase behavior of water near the liquidsolid transition in terms of the energy $E$ and temperature $T$. Red (blue) lines represent the liquid (solid) phases. The solid and dashed lines represent the stable and metastable phases. The vertical orange line is the liquid-solid coexistence phase. In the canonical ensemble, the energy $E=E(T)$ is determined by minimizing the free energy at each fixed $T$, while in the microcanonical ensemble, $T=T(E)$ is determined by maximizing the entropy at each fixed $E$. 

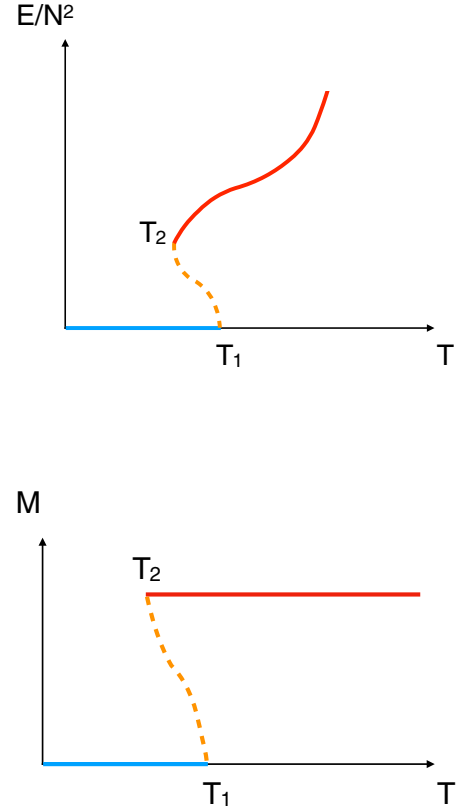

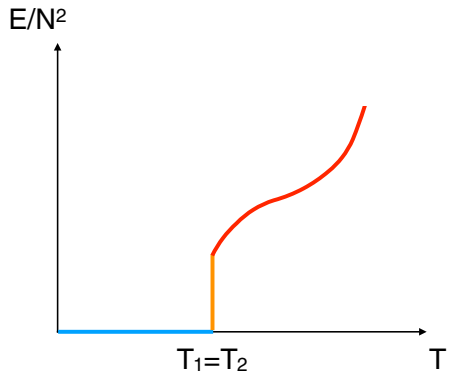

(a)

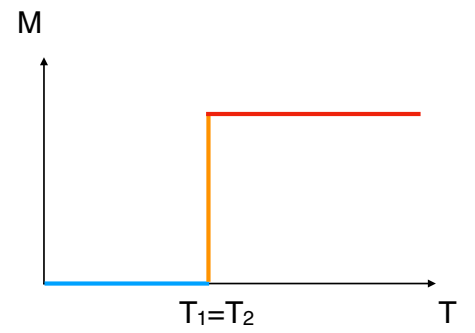

(b)
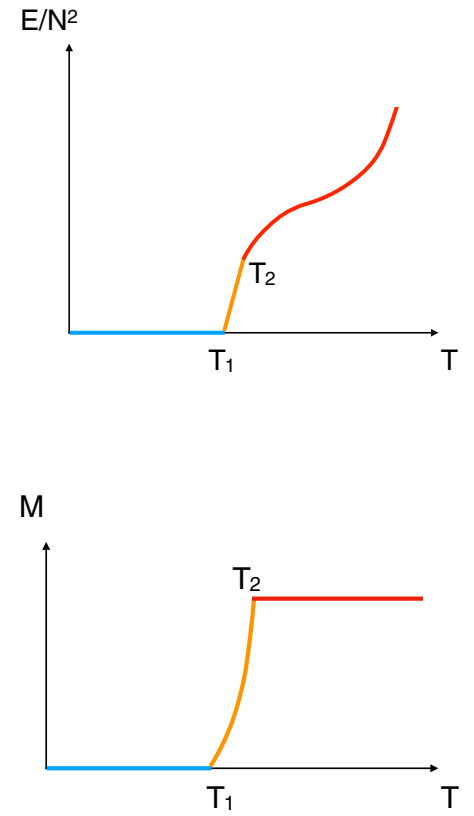

FIG. 2. The blue (red) lines represent the confined (deconfined) phases. Along the orange line is the partially deconfined coexistence phase. Similar figures have been used in Ref. [3]. (a) Three basic types of energy-vs-temperature relations in various large- $N$ gauge theories. (b) Three types of $M$-vs-temperature relations in various large- $N$ gauge theories. Here $M$ characterize the size of the deconned phase in the color space, namely $\mathrm{SU}(M)$ subgroup of $\mathrm{SU}(N)$ is deconned.

confusing, it will serve as a useful analog for what is happening in various phase transitions in later sections.

Two phases can coexist in the internal space. At the critical temperature $T=T_{\mathrm{c}}=0{ }^{\circ} \mathrm{C}$, water goes through a first-order transition, and the liquid and solid phases can coexist in physical space. The coexistence curve in the phase diagram is represented by the vertical orange line in Fig. 1. In a similar manner, two phases (e.g., confined and deconfined phases) can coexist in the internal space; see Figs. 2(a) and 2(b). The generalization of this idea to other theories with similar all-to-all couplings is straightforward.

Partial symmetry breaking can occur. Associated with the coexistence of two phases in the internal space, the symmetry of the system can be broken spontaneously. For example, $\mathrm{SU}(N)$ symmetry of gauge theory spontaneously breaks to ${ }^{1} \mathrm{SU}(M) \times \mathrm{SU}(N-M) \times \mathrm{U}(1)$. Moreover, the symmetry changes gradually; namely, at large $N$, the ratio $\frac{M}{N}$ changes continuously from 0 to 1 ; see Fig. 2(b). There is no counterpart to this phenomenon in the coexistence in physical space.

Temperature can change nontrivially. Ignoring the interactions at the interface of the two phases, the admixture of liquid and solid water in physical space determines the energy of the configuration at fixed temperature. On the other hand, temperature can change nontrivially, as shown

\footnotetext{
${ }^{1}$ Strictly speaking, the global part of gauge symmetry can break spontaneously. For details, see Ref. [4].
}

in the left and right panels of Fig. 2(a). The same can hold for other parameters, for example, when we replace the energy and temperature with charge and chemical potential.

"Metastable" phases can be completely stabilized in the thermodynamic limit. Associated with the first-order transition, metastable phases such as supercooled liquid and superheated solid can appear. Such metastable phases due to the local interaction are unstable to a small perturbation, even in the thermodynamic (large-volume) limit. On the other hand, similar metastable phases due to the all-to-all couplings can be stabilized in the thermodynamic limit (the large- $N$ limit); namely, the size of the perturbation needed to destabilize such phases can increase with the system size. While this fact has been well known for the large- $N$ gauge theories, the apparent difference from the local interaction has not been widely appreciated.

Previously, the features above have been conjectured for the confinement/deconfinement transition. In the rest of the paper, we argue that these features are more generically realized. To do so, we collect evidence based on concrete examples and heuristic arguments which apply to generic cases. We will start with qualitative arguments in a bound state of D-branes in Sec. II. The purpose of this section is to rephrase the statements in Refs. $[3,4]$ in a more intuitive manner, which will allow for more straightforward generalization beyond simple toy models. Following that discussion, we will outline a heuristic proposal for extending the lessons learned from the qualitative analysis to more 
generic cases. We will see in subsequent, varied examples that application of the basic ideas in our proposal can proceed without significant modification. In Sec. III, we will study QCD-like theories on a compact physical space (i.e., $\mathrm{S}^{3}$ ) in various limits giving both analytic and numerical quantitative evidence for the appearance of partialsymmetry-breaking phase transitions, and at the end, we will explore the possible effects that partial deconfinement could have on chiral symmetry. In Sec. IV, we will analyze partial-symmetry-breaking phase transitions in twodimensional (2D) pure Yang-Mills (YM) theories in both lattice and continuum formulations. In Sec. V, we will try to understand partial-symmetry-breaking phase transitions through gauge/gravity duality in the context of black hole/black string transitions and their relation to maximally supersymmetric 2D super-Yang-Mills (SYM) theory. In Sec. VI, the Higgsing in QCD with adjoint quarks is discussed. Finally, we will provide a brief recap, discussion, and outlook for future work.

\section{REVIEW AND QUALITATIVE ANALYSIS}

In this section, we will motivate our analysis with a discussion of a heuristic model that captures the broad features of the physics of phase transitions at large- $N$ that we wish to argue are universal. We will review key results in Ref. [3] in such a way that allows for straightforward generalization to other cases. The readers only interested in the derived intuition to be applied in more quantitative settings can skip to Sec. II B. For readers interested in further arguments for the universality of the large- $N$ phenomena explored below, see Appendix for a model of ant trail formation ${ }^{2}$ in a large colony population limit that behaves remarkably similarly to the brane model.

\section{A. Brane model}

Let us consider the world volume theory of $N$-coincident D-branes [5], which has a low-energy description as SYM theory with an $\mathrm{SU}(N)$ gauge symmetry [6]. The dynamics of the low energy world volume gauge theory are local with all-to-all coupling of the color degrees of freedom. Now, suppose we separate out and form a bound state of $M<N$ D-branes, i.e., a black hole. Due to the open string excitations, the extensive variables, e.g., entropy and energy, of the bound state are of order $\mathcal{O}\left(M^{2}\right)$.

Bringing another single D-brane near to the bound state excites $M$ different open string modes, giving rise to an $\mathcal{O}(M)$ attractive entropic force. If this additional brane falls into the black hole, the bound state energy and entropy increase as $E, S \sim M^{2} \rightarrow(M+1)^{2}$. However, due to $e^{-E / T}$ Boltzmann suppression, the more favorable state tends to

\footnotetext{
${ }^{2}$ In this case, since the ants are treated as indistinguishable, the symmetry group governing the dynamical process of trail formation within a colony of $N$ ants is the permutation group $S_{N}$.
}

have lower $M$. Thus, the size of the bound state is determined where the entropic force and Boltzmann suppression balance. In this way, two phase coexistence is possible in the equilibrium state of $M$ D-branes inside and $N-M$ D-branes outside the bound state.

In the large- $N$ limit, $M / N$ changes continuously. In the bound state phase, the world volume gauge symmetry is broken to $\mathrm{SU}(M) \times \mathrm{SU}(N-M) \times \mathrm{U}(1){ }^{3,4}$ In this way, partial symmetry breaking can occur. There are two phase transitions, $M / N=0$, where the bound state formation begins, and $M / N=1$, where all of the branes have fallen behind the horizon.

Let us make the argument more precise by considering the above thermodynamical system in the canonical ensemble with energy $E$ and temperature $T$. The partition function can be written as

$$
Z(T)=\int d E e^{-F(E, T) / T},
$$

where $F(E, T)=E-T S(E)$ is Helmholtz free energy. Extremizing $F$ with respect to $E$ and using the definition of the temperature in the microcanonical ensemble, $T_{\text {micro }}^{-1}:=d S / d E$, one can easily see that $\partial F / \partial E=0$ is equivalent to setting $T=T_{\text {micro }}$. Therefore, by finding the saddle points for fixed canonical temperature $T$, we obtain the energy $E$ which corresponds to the microcanonical temperature.

The stable $\left(\partial^{2} F / \partial E^{2}>0\right)$ or unstable $\left(\partial^{2} F / \partial E^{2}<0\right)$ saddles can equivalently be characterized by states with positive or negative specific heat, respectively. That is,

$$
\frac{\partial^{2} F}{\partial E^{2}}=\frac{T}{T_{\text {micro }}^{2}} \cdot \frac{d T_{\text {micro }}}{d E} .
$$

Figures 2(a) and 2(b) show a cartoon of three basic patterns of the phase diagram $[3,7,8]$ with the intermediate phase represented by the orange line. In the left (right) panel in each figure, the specific heat of the intermediate phase is negative (positive). The middle panel in each figure corresponds to the weak-coupling limit of pure YangMills theory $[7,8]$ where the saddle corresponding to the intermediate phase has $\partial^{2} F / \partial E^{2}=0$ regardless of the value of $E$.

The reason that nontrivial temperature dependence appears in the intermediate phase can be understood as follows [1,9]: roughly speaking, temperature is energy per number of dynamical degrees of freedom, and hence $T \sim E / M^{2}{ }^{5}$ Note that $M^{2}$ is parametrically determined

\footnotetext{
${ }^{3}$ If the flat direction is allowed, $\mathrm{SU}(M) \times \mathrm{U}(1)^{N-M-1}$ can also be realized.

${ }^{4}$ The meaning of the symmetry breaking will be elaborated on later in this paper.

${ }^{5}$ In the brane model, the energy is dominated by the contribution from the bound state.
} 
as a function of $E$, and hence the specific heat can be positive or negative depending on the details of the dynamics. In this way, temperature can change nontrivially.

When the phase diagram in the canonical ensemble is like the left panels of Figs. 2(a) and 2(b), the transition becomes of first order with hysteresis. Typically, free energies of two stable saddles are not equal, and hence one of them is just metastable. However, in order for the tunneling to happen, it is necessary to go beyond the unstable saddle, and hence a very large perturbation is needed. That is, the value of the size of the bound state $M$ and free energy $F$ have to change the amount of $\mathcal{O}(N)$ and $\mathcal{O}\left(N^{2}\right)$, respectively, and the tunneling rate becomes approximately $e^{-N^{2}}$. In this way, the metastable phase can be completely stabilized in the thermodynamic limit (large-N limit).

\section{B. Underlying mechanism}

Having constructed a rather simple model exhibiting partial-symmetry-breaking phase transitions, let us understand the mechanism behind the phase behavior and extract the lessons applicable to other systems as well.

Returning to the liquid/solid transition of water explained in Sec. I, the two phases have different energy due to finite latent heat. In the microcanonical ensemble, there is no discontinuity because the energy of the entire system and the volume occupied by each phase can continuously change in a correlated manner. As we will see below, essentially the same mechanism works for the degrees of freedom on the internal space.

Typically in the large- $N$ limit, $\mathcal{O}(N)$ degrees of freedom on the internal space have all-to-all interactions defined by the symmetries of the theory. Further, in the large- $N$ limit, phase transitions exhibit a large jump in the extensive thermodynamic variables in the canonical ensemble; e.g., the energy or charge changes significantly. In the microcanonical description, however, one expects that there is path in phase space describing a (possibly unstable) intermediate phase smoothly connecting the end points of the jump. The partially broken symmetries in the theory at points on the path through the intermediate phase then smoothly interpolate between the symmetries in the (meta) stable phases, e.g., the $\mathrm{SU}(M) \times \mathrm{SU}(N-M) \times \mathrm{U}(1)$ phase for the D-brane picture of black hole formation or the $\mathrm{S}_{M} \times \mathrm{S}_{N-M}$ phase in the ant model in Appendix.

This raises a question in the intermediate phase: what if, e.g., all $N$ D-branes formed a bound state (or all $N$ ants formed a trail) immediately at the phase transition, and all degrees of freedom on the internal space were excited simultaneously at small energy? In this case, the temperature $T \sim E / N^{2}$ would have to become parametrically small, and hence the microcanonical phase diagram would have a discontinuity. Therefore, as a function of $E$, the number of unlocked degrees of freedom $M^{2}$ has to increase gradually in such a way that $E / M^{2}$ is continuous. ${ }^{6}$ A similar way to phrase it is that in quantum mechanics it is impossible to excite all degrees of freedom in a parametrically small amount on a compact space because each mode is quantized. In the case of liquid/solid transition of water, the latent heat functions similarly to suppress parametrically small excitations of the entire system.

Considering the case of a theory with an $\mathrm{SU}(N)$ symmetry group with large $N$, the continuous change of the symmetry cannot generically be surmised simply from the continuity of the temperature alone. Although the excitation of $M^{2}$ degrees of freedom will break the full $\mathrm{SU}(N)$ symmetry, the system in this state still tends to exhibit a large symmetry. We conjecture this because it is natural to expect that the saddles (the maximization of the entropy in the case of the microcanonical ensemble) respect the symmetry as much as possible. In the case of confinement/deconfinement transition, the precise understanding is obtained in Ref. [10], by using a close relationship to Bose-Einstein condensation [11].

Essentially the same can happen in other systems as well. The point is that there are many internal degrees of freedom interacting, and they can be separated to two phases in the internal space. The continuity in the microcanonical ensemble requires the gradual change of the numbers of degrees of freedom in two phases. The same principle naturally leads to the intermediate phase, or equivalently the coexistence of two phases. Furthermore, when the system has a large symmetry, it is natural to expect the continuous change of the symmetry.

An important difference is how $M$ in the nonlocal theories ${ }^{7}$ - or the volume of each phase in the local theories-depends on the energy $E$. In the local theories, as long as the contribution from the interface is negligible, the volume depends linearly on the energy, analogous to the center panel of Fig. 2(b). In the nonlocal theories, more complicated behavior can show up.

When the specific heat of the intermediate phase is negative, the intermediate saddle becomes the maximum of free energy in canonical ensemble, which separates stable and metastable phases. The amount of fluctuation needed for the tunneling to the other (meta)stable branches increases with $N$. Therefore, in the thermodynamic limit (large- $N$ limit), the probability of seeing a tunneling event becomes vanishingly small, and the metastable state is completely stabilized at $N=\infty$.

\footnotetext{
${ }^{6}$ One may think the continuity can be achieved if a small fraction in the spatial volume goes thorough complete deconfinement. However, note that the deconfinement takes place even in matrix models, in which there is no spatial dimension at all. See Sec. II B 1 for more explanation regarding this issue.

${ }^{7}$ As noted in Sec. I, the internal space consisting of the color degrees of freedom can be regarded as a nonlocal space, in the sense that the degrees of freedom on $N^{2}$ points have all-to-all interaction.
} 
All of the ingredients explained above are not specific to the deconfinement transition in gauge theory, and so it is a reasonable conjecture that there is a large universality class of theories that exhibit such phase behavior.

\section{Large volume vs small volume}

The thermodynamic limit can be realized by sending the volume of the physical space to infinity or by taking the size internal space to be infinity (large- $N$ limit). In the large- $N$ limit, the physical volume is so small that the coexistence of two phases in physical space is impossible, we need to consider only the coexistence in the internal space. What if both internal and physical spaces are large?

Let us consider microcanonical ensemble, and suppose we prepare an initial state in the intermediate phase with uniform energy density. Locally, there are three basic patterns as we have already seen [Figs. 2(a) and 2(b)], as in the case of the small volume. If the specific heat in the intermediate phase is negative, fluctuations in the internal space degrees of freedom can lead to instability in the physical space. ${ }^{8}$ Typically, a spinodal decomposition takes place, and most of the physical space is occupied by the phases with either $M / N=0$ or $M / N=1$. Yet, the intermediate phase $0<M / N<1$ survives on the domain wall separating regions existing in the two (meta)stable phases. On the other hand, if the specific heat coming from the dynamics in the internal space is positive, then such instability does not arise, and the intermediate state can stably fill whole the physical space.

Let us consider further on the case with the negative specific heat [the left panels of Figs. 2(a) and 2(b)]. Treating the system now in the canonical ensemble, the phase with either $M / N=0$ or $M / N=1$ filling whole the physical space gives the minimum of free energy. However, depending on the temperature, these saddles might be only local minima. Whether tunneling to the global minimum takes place or not depends on the order of limits in taking both large- $N$ and large physical volume; that is, fixing $N$ large but finite and taking a large-volume limit may see different tunneling rates as compared to the limit taking large but fixed volume and $N \rightarrow \infty$. For example, for a given region at fixed volume in physical space, taking the large- $N$ limit parametrically suppresses the probability of any tunneling to occur, e.g., tunneling rates in the gauge theories are typically $\mathcal{O}\left(e^{-N^{2}}\right)$ suppressed; however, in fixing $N$ large but finite, the large physical volume limit

\footnotetext{
${ }^{8}$ Consider two small neighboring volumes $A$ and $B$ in thermodynamic equilibrium and a small amount of energy moved from $A$ to $B$. After the energy transfer, the temperature in $A$ will be slightly higher than that in $B$ because the specific heat is negative. Following the energy transfer, the resulting gradient of the temperature induces further energy transfer, and thus, after a small perturbation, the difference of the temperatures in regions $A$ and $B$ grows.
}

will eventually surpass the large- $N$ suppression and allow for a non-negligible tunneling probability.

\section{Gauge-invariant description and spontaneous gauge symmetry breaking}

We have seen that the $\mathrm{SU}(N)$ symmetry of the brane model is "spontaneously broken" to $\mathrm{SU}(M) \times \mathrm{SU}(N-M) \times$ $\mathrm{U}(1)$. Let us elaborate on this point and identify the order parameter $[4,10]$.

First, recall that the physical states in the Hilbert space have to be gauge invariant. However, the discussion above does not seem to be consistent with the gauge invariance. We can easily fix it and understand everything in the gaugeinvariant manner, in the following way [4]. D-branes and open strings are described by using the $N \times N$ matrices. First, we consider a particular embedding of $\mathrm{SU}(M)$ into $\mathrm{SU}(N)$ (approximately a particular choice of $M$ D-branes), e.g., the upper-left $M \times M$ block as in the top panel of Fig. 3. Then, by considering the excitation in this particular $\mathrm{SU}(M)$ embedding, we are probing the space of $\mathrm{SU}(M)$ invariant states denoted $|E ; \mathrm{SU}(M)\rangle$. Note that by construction the states $|E ; \mathrm{SU}(M)\rangle$ are not $\mathrm{SU}(N)$ invariant. From these $\mathrm{SU}(M)$-invariant states, we can construct the $\mathrm{SU}\left(N_{c}\right)$-invariant states with the same energy as

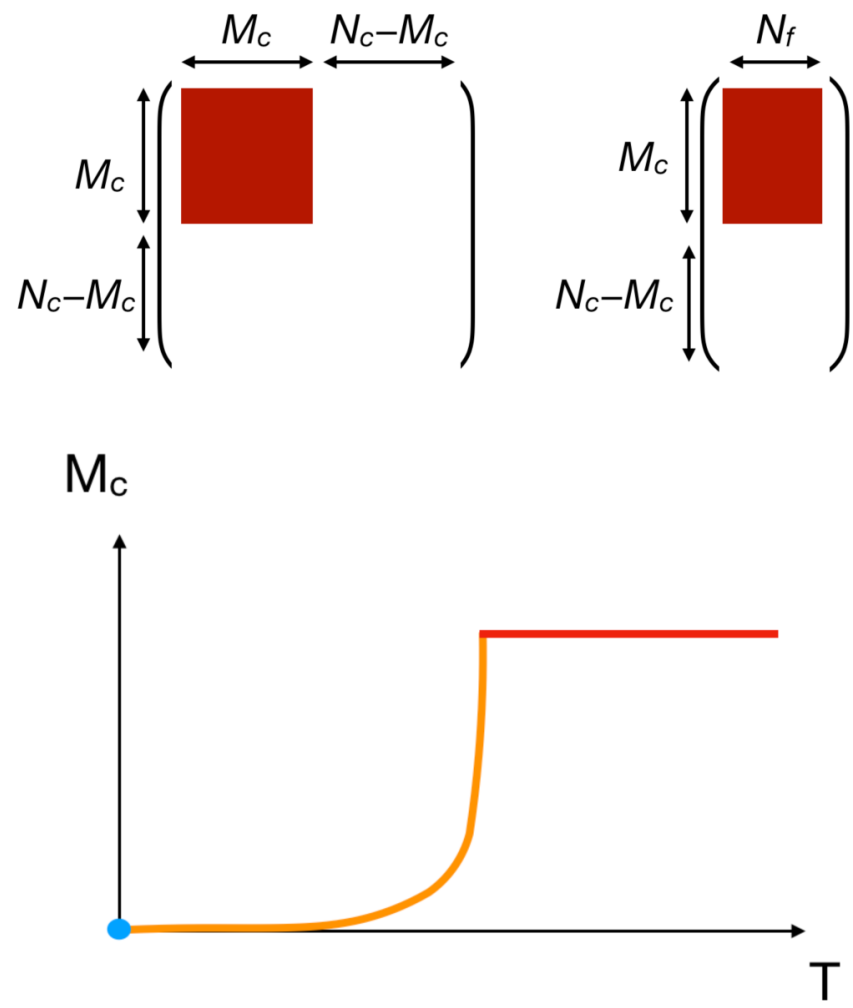

FIG. 3. Above is a sketch of partial deconfinement in the weakly coupled QCD. In the gauge sector, the $\mathrm{SU}\left(M_{c}\right)$ block is deconfined, while the rest is confined. In the quark sector, $M_{c}$ colors of all $N_{f}$ flavors are deconfined. Complete confinement is realized only at $T=0$. 


$$
|E\rangle_{\mathrm{inv}}=\mathcal{N}^{-1 / 2} \int d U \mathcal{U}(|E ; \mathrm{SU}(M)\rangle) .
$$

This mapping is one to one. These $\mathrm{SU}(N)$-invariant states are the partially deconfined states which dominate thermodynamics.

Next, let us see how the $\mathrm{SU}(N)$ symmetry is spontaneously broken to $\mathrm{SU}(M) \times \mathrm{SU}(N-M) \times \mathrm{U}(1)$. To start, let us consider two different embeddings of $\mathrm{SU}(M)$ into $\mathrm{SU}(N)$ (approximately two different choices of $M$-branes). We denote the $\mathrm{SU}(M)$-invariant states for the respective embeddings by $|E ; \mathrm{SU}(M)\rangle_{1}$ and $|E ; \mathrm{SU}(M)\rangle_{2}$. Further, let us consider operators $\hat{O}$ that affect at most an $O\left(N^{0}\right)$ change the energy, e.g., $\hat{O}$ constructed as the trace of a product of $O\left(N^{0}\right)$ number of scalars. That is, because we are interested in the properties of the states of energy $O\left(N^{2}\right)$, we do not want to consider operator insertions that could change the energy too much. For such operators, we have

$$
{ }_{1}\langle E ; \mathrm{SU}(M)|\hat{O}| E ; \mathrm{SU}(M)\rangle_{2}=0 .
$$

Note that $\frac{M}{N}$ can be small but order $O\left(N^{0}\right)$. The vanishing (4) is due to the fact that in order to connect the two states $O\left(N^{2}\right)$ creation and annihilation operators are needed. This is analogous to the superselection; $|E ; \mathrm{SU}(M)\rangle$ and $|E\rangle_{\text {inv }}$ defined by (3) are indistinguishable in the sense that we get the same expectation value. More precisely in a very small spatial region, different embeddings can be connected without changing the energy too much, but globally, different embeddings cannot be connected. Essentially, this is the spontaneous symmetry breaking of the global part of the gauge symmetry.

In Yang-Mills theory, the value of $M$ is directly visible in the distribution of the phases of Polyakov loop $\rho(\theta)$, in a gauge-invariant manner [10]. In the next section, we will see this point in a few concrete examples.

\section{QCD PHASE TRANSITION}

In this section, we will consider the coexistence of confined and deconfined phases in large- $N_{c}$ QCD with the $\mathrm{SU}\left(N_{c}\right)$ gauge group and $N_{f}$ quarks in two different limits. First, we will see that, in the analytically solvable regime of weakly coupled large- $N_{c}$ QCD on a small $\mathrm{S}^{3}$, an explicit construction of the partial deconfinement transition is possible. Second, we will study the consequences of partial deconfinement for chiral symmetry in the infinitevolume limit of large- $N_{c}$ QCD with $N_{f}$ massless quarks.

\section{A. Weakly coupled QCD on $\mathbf{S}^{\mathbf{3}}$}

Let us consider $\mathrm{SU}\left(N_{c}\right)$ YM with $N_{f}$ fermions of mass $m$ in the fundamental representation of the gauge algebra $\mathfrak{s} \mathfrak{t}\left(N_{c}\right)$ and take large $N_{c}$ with $\frac{N_{f}}{N_{c}}$ fixed. We will put this theory on $S^{3} \times S^{1}$ where the $S^{3}$ radius is $R$ and $S^{1}$ radius is $\beta$, the weak-coupling limit can be solved analytically in the regime where $\frac{1}{R} \gg \Lambda_{\mathrm{QCD}}[7,8,12,13]$.

$$
\text { 1. } N_{f}=0
$$

Because $N_{f}>0$ is technically complicated, let us start with a simpler case setting $N_{f}=0$, which has been studied in Ref. [4]. After understanding the $N_{f}=0$ case, the generalization to $N_{f}>0$ is more tractable. To study the analytically solvable regime further, let us fix the gauge so that the temporal component of gauge field $A_{t}$ is static and diagonal. In this gauge, all of the spatial components of the gauge field can be integrated out, leaving behind an effective action for $A_{t}$ in terms of $u_{n}=\frac{1}{N} \operatorname{Tr} \mathcal{P}^{n}$. Here, we have denoted the Polyakov line made of $A_{t}$ as $\mathcal{P}$. The $u_{n}$ 's are determined such that the effective action is minimized. The minimum of the effective action is interpreted as $\beta F$, where $F$ is the free energy of the theory and $\beta=1 / T$ is the inverse temperature. We use the same notation $\beta F$ for both the effective action and free energy, with the understanding that the former is the function of $u_{n}$ 's and the latter is obtained by taking the saddle point value. Explicitly computing the effective action, one obtains the effective action

$$
\beta F=N_{c}^{2} \sum_{n} a_{n}(T) u_{n}^{2}
$$

where

$$
a_{n}(T)=\frac{1}{n}\left(1-2 \sum_{l=1}^{\infty} l(l+2) e^{-n \frac{\beta(l+1)}{R}}\right) .
$$

In the following, we will be concerned primarily with the thermodynamic profile of loop operators built out of $\mathcal{P}$. The phase distribution of the Polyakov line is given by

$$
\rho(\theta)=\frac{1}{2 \pi}\left(1+2 \sum_{n} u_{n} \cos (n \theta)\right),
$$

where $-\pi \leq \theta<\pi$. At low temperature, all $a_{n}$ 's are positive, and hence all $u_{n}$ 's take zero. This is the confining phase, $\rho(\theta)=\frac{1}{2 \pi}$. The phase transition happens at $T=T_{c}$, where $a_{1}$ becomes zero:

$$
a_{1}\left(T_{c}\right)=0 .
$$

At this point, other $a_{n}$ 's $(n \geq 2)$ are still positive. Therefore, $u_{1}$ can take any value without changing free energy, while other $u_{n}$ 's remain zero, as long as $\rho(\theta) \geq 0$ is not violated. The distribution of the Polyakov line phase becomes

$$
\rho(\theta)=\frac{1+2 u_{1} \cos \theta}{2 \pi}=\frac{1+2 P \cos \theta}{2 \pi},
$$


where $P=\frac{1}{N} \operatorname{Tr} \mathcal{P}$, and hence $u_{1}=P$ can take any value between 0 and $\frac{1}{2}$. Above $T_{c}$, other $u_{n}$ 's take nonzero values as well, such that the effective action is minimized without violating $\rho(\theta) \geq 0$.

The energy at $T=T_{c}$ is ${ }^{9}$

$$
E=N_{c}^{2} P^{2} \times\left.\frac{\partial a_{n}}{\partial \beta}\right|_{T=T_{c}} .
$$

The entropy is obtained as $S=\beta(E-F)$, which is simply $\beta E$ at $T=T_{c}$.

Therefore, the phase diagram becomes like the center panels of Figs. 2(a) and 2(b). In the microcanonical ensemble, there are two transitions, at $P=0$ and $P=\frac{1}{2}$. The latter is the Gross-Witten-Wadia (GWW) transition $[14,15]$, which corresponds to the formation of the gap in the phase distribution.

We identify the GWW transition with the transition from partially deconfined phase to completely deconfined phase. The energy and entropy of the $\mathrm{SU}\left(M_{c}\right)$-deconfined phase should be those of the $\mathrm{SU}\left(M_{c}\right)$ theory at the GWW point $[1,3]$ :

$$
\begin{aligned}
& S=S_{\mathrm{GWW}}\left(M_{c}\right), \\
& E=E_{\mathrm{GWW}}\left(M_{c}\right) .
\end{aligned}
$$

These relations actually hold, with the following identification:

$$
P=\frac{M_{c}}{2 N_{c}}
$$

Another nontrivial relation is obtained by looking at the distribution of Polyakov loop phases. Because $N_{c}-M_{c}$ of the phases are in the confining phase while the other $M_{c}$ are at the GWW point of the $\mathrm{SU}(M)$ theory, we naturally expect [3]

$$
\begin{aligned}
\rho(\theta) & =\left(1-\frac{M_{c}}{N_{c}}\right) \rho_{\text {confine }}(\theta)+\frac{M_{c}}{N_{c}} \cdot \rho_{\mathrm{GWW}}\left(\theta ; M_{c}\right) \\
& =\frac{1}{2 \pi}\left(1-\frac{M_{c}}{N_{c}}\right)+\frac{M_{c}}{N_{c}} \cdot \rho_{\mathrm{GWW}}\left(\theta ; M_{c}\right) .
\end{aligned}
$$

Here, $\rho_{\mathrm{GWW}}\left(\theta ; M_{c}\right)$ is the distribution in the $\mathrm{SU}\left(M_{c}\right)$ theory at the GWW point. This relation holds as well, with the identification (13) and $\rho_{\mathrm{GWW}}\left(\theta ; M_{c}\right)=\frac{1+\cos \theta}{2 \pi}$.

We can push the argument further and demonstrate partial deconfinement more robustly by constructing the

\footnotetext{
${ }^{9}$ By definition, $E=-\partial(\beta F) / \partial \beta$. In order to use this, we need to introduce a small interaction so that the $\beta$-derivative is mathematically well defined. For example, we can use the expression for nonzero $\frac{N_{f}}{N_{c}}(19)$ and then send $\frac{N_{f}}{N_{c}}$ to zero.
}

states in the Hilbert space [4]. The argument is similar to the one provided in Sec. II B 2.

$$
\text { 2. } N_{f}>0
$$

Let us move on to the case of $N_{f}>0$. The effective action is $[12,13]$

$$
\beta F=\sum_{n}\left(N_{c}^{2} a_{n}(T) u_{n}^{2}+N_{c} N_{f} b_{n}(T) u_{n}\right),
$$

where $a_{n}$ is given by (6), and

$$
b_{n}(T)=\frac{(-1)^{n}}{n} \cdot 4 \sum_{l=1}^{\infty} l(l+1) e^{-n \frac{\beta}{R} \sqrt{\left(l+\frac{1}{2}\right)^{2}+m^{2} R^{2}}} .
$$

In the following, we will be concerned primarily with the thermodynamic profile of loop operators built out of $\mathcal{P}$ again. By definition, the phase distribution of the Polyakov loop given in (7) must be positive semidefinite: $\rho(\theta) \geq 0$. Further in the low-temperature regime $\beta \gg R$, the phase distribution $\rho(\theta)$ is everywhere nonzero. However, there is a GWW phase transition, which we denote by $T=T_{\mathrm{GWW}}$, above which $\rho(\theta)$ is zero for a part of the range of $\theta$.

As long as this phase distribution is everywhere positive, $u_{n}$ 's can change independently, and the saddle point equation $\frac{\partial(\beta F)}{\partial u_{n}}=0$ can be solved for each $n$ as

$$
u_{n}=-\frac{N_{f}}{N_{c}} \cdot \frac{b_{n}(T)}{2 a_{n}(T)} .
$$

Evaluating the effective action on the saddle, i.e., substituting (17) into (15), we obtain

$$
\beta F=-\frac{N_{f}^{2}}{4} \sum_{n} \frac{\left(b_{n}(T)\right)^{2}}{a_{n}(T)} .
$$

From this point, computing the internal energy of the thermodynamic system $\left(E=-\frac{\partial(\beta F)}{\partial \beta}\right)$ yields

$$
\begin{aligned}
E & =\frac{N_{f}^{2}}{4} \sum_{n}\left\{\left(\frac{b_{n}}{a_{n}}\right)^{2} \cdot \frac{\partial a_{n}}{\partial \beta}-2 \frac{b_{n}}{a_{n}} \cdot \frac{\partial b_{n}}{\partial \beta}\right\} \\
& =\sum_{n}\left(N_{c}^{2} u_{n}^{2} \frac{\partial a_{n}}{\partial \beta}+N_{c} N_{f} u_{n} \frac{\partial b_{n}}{\partial \beta}\right) .
\end{aligned}
$$

Note that the expressions (17), (18), and (19) are valid only for $T \leq T_{\mathrm{GWw}}$.

At this point, we need to demonstrate that partial deconfinement is actually taking place at a temperature $T \leq T_{\mathrm{GWW}}$. To begin, consider the situation that $M_{c}$ colors and $N_{f}$ flavors are deconfined, as shown in Fig. 3. Corresponding to a given temperature $T$, we identify the size of the deconfined block $M_{c}$ such that $T$ is the 
GWW-transition temperature of the $\mathrm{SU}\left(M_{c}\right)$ theory, which we denote $T=T_{\mathrm{GWw}}\left(M_{c}, N_{f}\right)$.

We start by computing free energy $\tilde{F}$ for an $\mathrm{SU}\left(M_{c}\right)$ YM theory with $N_{f}$ fundamental fermions of mass $m$. This is trivially obtained by replacing $N_{c}$ in (15) by $M_{c}$,

$$
\beta \tilde{F}=\sum_{n}\left(M_{c}^{2} a_{n}(T) \tilde{u}_{n}^{2}+M_{c} N_{f} b_{n}(T) \tilde{u}_{n}\right) .
$$

Here, for convenience in distinguishing variables, we have expressed the phase distribution of the Polyakov line in the $\mathrm{SU}\left(M_{c}\right)$ theory as

$$
\tilde{\rho}(\theta) \equiv \frac{1}{2 \pi}\left(1+2 \sum_{n} \tilde{u}_{n} \cos (n \theta)\right) .
$$

We can now determine the GWW temperature by solving

$$
\min \tilde{\rho}(\theta)=0 .
$$

By identifying $\tilde{u}=\frac{N_{c}}{M_{c}} u$, we can trivially rewrite (20) to (15), while (22) changes to

$$
\min \rho(\theta)=\frac{1}{2 \pi}\left(1-\frac{M_{c}}{N_{c}}\right),
$$

where again $\rho(\theta)$, given in (7), is the phase distribution of the Polyakov line in the $\mathrm{SU}\left(N_{c}\right)$ theory. It is easy to see that the key relation (14) is actually satisfied. This relation (23) gives a simple way to identify $M_{c}$ for a given temperature. See Fig. 3 for a qualitative picture for the temperature dependence.

Further, computing the energy $E$ in the $\mathrm{SU}\left(M_{c}\right)$ theory is straightforward and results in

$$
\begin{aligned}
E & =\sum_{n}\left(N_{c}^{2} u_{n}^{2} \frac{\partial a_{n}}{\partial \beta}+N_{c} N_{f} u_{n} \frac{\partial b_{n}}{\partial \beta}\right) \\
& =\sum_{n}\left(M_{c}^{2} \tilde{u}_{n}^{2} \frac{\partial a_{n}}{\partial \beta}+M_{c} N_{f} \tilde{u}_{n} \frac{\partial b_{n}}{\partial \beta},\right),
\end{aligned}
$$

and the entropy is $S=\beta(E-F)$ and is easily obtained from the above expressions. By substituting $T=$ $T_{\mathrm{GWw}}\left(M_{c}, N_{f}\right)$, we obtain

$E=E_{\mathrm{GWW}}\left(M_{c}, N_{f}\right), \quad$ and $\quad S=S_{\mathrm{GWW}}\left(M_{c}, N_{f}\right)$.

Therefore, the energy and entropy behave consistently with (23).

The method of constructing a one-to-one mapping between the states in the Hilbert spaces of the $\mathrm{SU}\left(N_{c}\right)$ theory and the $\mathrm{SU}\left(M_{c}\right)$ theory, explained around (3), is completely applicable in the above analysis.

In the original treatment of this theory [12], the GWW transition has been identified with the deconfinement transition. The reasoning behind this identification is based on the observation that the free energy is of order $N_{f}^{2}$ below the GWW transition —as can be seen in (18) —and the argument that $N_{f}^{2}$ counts the number of mesonic degrees of freedom. However, as we have seen, the GWW transition should be identified with the deconfinement transition of all color degrees of freedom. Below this maximal GWW transition, there is a coexistence phase on the internal space, i.e., the group manifold of $S U\left(N_{c}\right)$, where confined and deconfined sectors are simultaneously realized for a complementary subset of the color degrees of freedom.

\section{B. Chiral symmetry}

In this section, our aim is to understand the fate of chiral symmetry as one moves into the partially deconfined phase. That is, does an intermediate phase connecting phases of broken and unbroken chiral symmetry exist? While there are no quantitative, dynamical calculations yet to determine the existence of such a coexistence phase, by exploiting a known loophole in the Vafa-Witten theorem [16], we will explore the possibility that an intermediate phase with partially broken chiral symmetry could exist.

Let us now consider the infinite-volume limit of large- $N_{c}$ $\mathrm{SU}\left(N_{c}\right)$ QCD with $N_{f}$ fermions in the fundamental representation. In order to preserve the chiral symmetry precisely, let us consider quarks in this theory to be exactly massless. We will write the massless quarks as $\psi_{c f}$ with the color and flavor indices taking values $c=1,2, \ldots, N_{c}$ and $f=1,2, \ldots, N_{f}$, respectively. If an intermediate phase connecting the confined phase and deconfined phase does exist, a naive guess as to its structure could be as follows:

(i) In the gauge sector, $\mathrm{SU}\left(M_{c}\right) \subset \mathrm{SU}\left(N_{c}\right)$ deconfines, and we assume that the symmetry breaking occurs in such a way that $c=1,2, \ldots, M_{c}$ spans the deconfined block as in the left panel of Fig. 4.

(ii) In the quark sector, only the massless modes $\psi_{c f}$ with $c=1,2, \ldots, M_{c}$ and $f=1,2, \ldots, M_{f}$ deconfine. That is, we have $M_{c}$ color and $M_{f}$ flavor degrees of freedom deconfined as depicted in the right panel of Fig. 4.

(iii) The left over $\left(N_{c}^{2}-M_{c}^{2}\right.$ degrees of freedom in the gauge sector and $N_{c} N_{f}-M_{c} M_{f}$ degrees of freedom in the quark sector) remain confined.

There are, however, immediate objections that could be raised if the above case is true. One should note above that the minimal flavor symmetry breaking pattern would involve the breaking of $S U\left(N_{f}\right)_{V}$. Such a flavor symmetry breaking patter would seem to be in violation of the VafaWitten theorem [16], which forbids the breaking of the vector part of the flavor symmetry. However, we can use a well-known exception: the Vafa-Witten theorem assumes the positivity of the fermion determinant in the Euclidean path integral, which is dependent on the value of the baryon chemical potential $\mu_{B}$. At $\mu_{B}=0$, the above assumption is valid, and hence we expect $M_{f}=N_{f}$, just as Fig. 3 . 


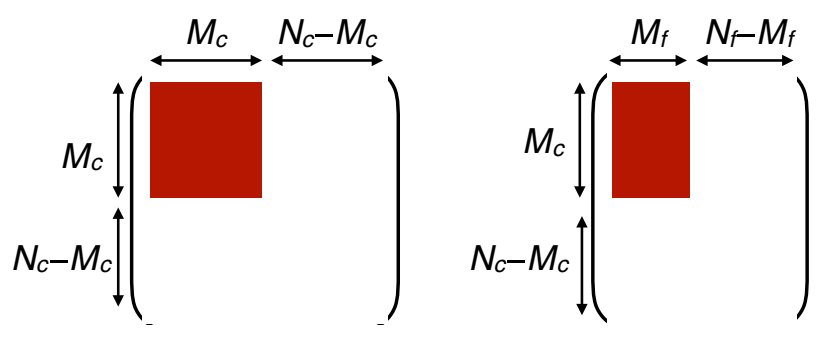

FIG. 4. In the gauge sector, the $\mathrm{SU}\left(M_{c}\right)$ block is deconfined, while the rest is confined. In the quark sector, $M_{c}$ colors of $M_{f}$ flavors are deconfined. The chiral symmetry is broken in the deconfined sector; namely, for $M_{c}$ colors, it breaks to $\mathrm{SU}\left(M_{f}\right)_{A}$, while for the other $N_{c}-M_{c}$ colors, it breaks completely. Based on the Vafa-Witten theorem [16], $M_{f}=N_{f}$ is expected at zero baryon chemical potential.

However, one can introduce $\mu_{B} \neq 0$ without breaking chiral symmetry, and because the fermion determinant is complex at finite baryon chemical potential, the Vafa-Witten theorem does not apply. Thus, $M_{f}<N_{f}$ at $\mu \neq 0$ may be possible.

If we can assume that the deconfinement pattern above holds, a natural expectation is that the chiral condensate is zero for $c=1,2, \ldots, M_{c}$ and $f=1,2, \ldots, M_{f}$. Then, in the $\mathrm{SU}\left(M_{c}\right)$ sector, $\mathrm{SU}\left(N_{f}\right)_{A}$ spontaneously breaks to $\mathrm{SU}\left(M_{f}\right)_{A}$, and $\mathrm{SU}\left(N_{f}\right)_{V}$ spontaneously breaks to $\mathrm{SU}\left(M_{f}\right)_{V} \times \mathrm{SU}\left(N_{f}-M_{f}\right)_{V}$. In the $\mathrm{SU}\left(N_{c}-M_{c}\right)$ sector, $\mathrm{SU}\left(N_{f}\right)_{A}$ breaks completely, and $\mathrm{SU}\left(N_{f}\right)_{V}$ does not break. Here, $M_{c}$ and $M_{f}$ are determined dynamically as the function of the energy. While we cannot analytically prove this statement, we can do a consistency check by using the 't Hooft anomaly, which is somewhat trivial.

In the usual sense of anomaly matching in the broken and unbroken chiral symmetry phases, treating the global chiral symmetry $\mathrm{SU}\left(N_{f}\right)_{A}$ as a background gauge symmetry, the anomaly is easily calculated in the UV to be proportional to $\operatorname{dim} \mathrm{SU}\left(N_{f}\right)=N_{f}^{2}-1$. If along the flow to the IR $\mathrm{SU}\left(N_{f}\right)_{A}$ is completely broken, the anomaly of the WessZumino-Witten action of the Nambu-Goldstone (NG) boson takes the same value.

However, the logic of anomaly matching should be satisfied at any intermediate energy scale, provided there are no accidental symmetries along the flow. Then, in the partially deconfined phase where there is an admixture deconfined and confined sectors coexisting, we should be able to perform the same counting as above. If the gauge symmetry and chiral symmetry break in a coordinated manner as we have conjectured above in the deconfined $\mathrm{SU}\left(M_{c}\right)$ sector, then the quarks contribute $M_{f}^{2}-1$, while the NG boson corresponding to the coset $\mathrm{SU}\left(N_{f}\right)_{A} /$ $\mathrm{SU}\left(M_{f}\right)_{A}$ contributes $N_{f}^{2}-M_{f}^{2}$. Thus, the sum is always $N_{f}^{2}-1$, and the anomaly in the partially deconfined phase matches the UV anomaly. In the confined $\mathrm{SU}\left(N_{c}-M_{c}\right)$ sector, the anomaly matching is trivially satisfied; the chiral symmetry is completely broken or unbroken.
By considering the chiral condensate for each flavor and color, possible breaking of the chiral symmetry and color symmetry can be detected. The distribution of the phases of Polyakov loop can also be used to see the "breaking" of the color symmetry.

As noted in the many caveats throughout this section, the breaking pattern we have discussed above is merely a logical possibility, and we are without the dynamical calculations needed in order to see if it is actually realized. It would be interesting to understand the effect of the nondegenerate quark mass on the flavor symmetry breaking pattern. Partial breaking of flavor symmetry may take place in other corners of the phase diagram as well, such as the hadron condensation at low temperature and large baryon chemical potential.

\section{PURE YANG-MILLS IN TWO DIMENSIONS}

In this section, we consider two solvable examples of twodimensional pure Yang-Mills theory in Euclidean spacetime at finite coupling: Wilson's plaquette action and the continuum theory on 2-sphere. While these theories have been solved analytically several decades ago and well studied since then, to our knowledge, the simple physical interpretation of their phase behavior that we will provide has not been appreciated. In these models, we will explicitly demonstrate that as the 't Hooft coupling $\lambda$ varies, a transition into a phase analogous to the coexistence phase of confined and deconfined degrees of freedom takes place. An important difference from the previous example is that these examples do not admit the Wick rotation to Minkowski spacetime, and thus we are not manifestly concerned about the interpretation of states in the Hilbert space. Rather, we focus our attention on the properties of the saddle points dominating the path integral in the large- $N$ limit. We obtain simple results consistent with our picture of partial-symmetry-breaking phase transitions, which supports our conjecture that such transitions are generic.

\section{A. Gross-Witten-Wadia transition in 2D lattice gauge theory}

The first model that we will consider is the two-dimensional version of Wilson's plaquette formulation of $\mathrm{U}(N)$ gauge theory on a square lattice. This theory has been solved at large $N$, and a third-order (GWW) transition has been found $[14,15]$. Historically, this model is, obviously, the first theory in which the GWW was found, the derivation of which goes as follows.

We start by fixing the axial gauge. That is, we enforce that the link variable connecting lattice sites along the time direction is taken to be the unit matrix. In the axial gauge, the partition function becomes

$$
Z=\left(\int d \mathcal{U} e^{-\frac{1}{g^{2}}\left(\operatorname{Tr} \mathcal{U}+\operatorname{Tr} \mathcal{U}^{\dagger}\right)}\right)^{V} \equiv z^{V},
$$


where $V$ is the number of lattice sites, the plaquette $\mathcal{U}$ is $N \times N$ unitary matrix, and the integral is taken with respect to the Haar measure. Therefore, the problem of computing the full path integral is reduced to simply computing the one-matrix model partition function $z$.

To solve this theory, let $\left\{e^{i \theta_{1}}, \ldots, e^{i \theta_{N}}\right\}$ denote the set of eigenvalues of $\mathcal{U}$ where the phases take values in $\theta_{i} \in$ $(-\pi, \pi]$ for all $i=1, \ldots, N$. The large- $N$ limit is taken with fixed $\lambda=g^{2} N$. In the large- $N$ limit, the one-matrix model can be solved by finding the saddle point expressed by the distribution of phases, $\rho(\theta)$, which has a nontrivial dependence on the coupling.

For $\lambda \geq 2$, the distribution of phases takes the form

$$
\rho(\theta)=\frac{1}{2 \pi}\left(1+\frac{2}{\lambda} \cos \theta\right) .
$$

However, in the regime where $\lambda<2$, the distribution has a markedly different profile in $\theta$,

$\rho(\theta)=\left\{\begin{array}{ll}\frac{2}{\pi \lambda} \cos \frac{\theta}{2} \sqrt{\frac{\lambda}{2}-\sin ^{2} \frac{\theta}{2}} & \left(|\theta| \leq 2 \arcsin \sqrt{\frac{\lambda}{2}}\right) \\ 0 & \left(|\theta|>2 \arcsin \sqrt{\frac{\lambda}{2}}\right)\end{array}\right.$.

The free energy $F=-\log z$ near $\lambda=2$ in the two distinct regimes described above displays a phase transition:

$$
\frac{F}{N^{2}}=\left\{\begin{array}{ll}
-\frac{1}{\lambda^{2}} & (\lambda \geq 2) \\
-\frac{2}{\lambda}+\frac{1}{2} \log \left(\frac{2}{\lambda}\right)+\frac{3}{4} & \lambda<2
\end{array} .\right.
$$

One can immediately deduce the existence of a third-order phase transition at $\lambda=2$ insofar as at the transition point $F$, $\frac{d F}{d \lambda}$ and $\frac{d^{2} F}{d \lambda^{2}}$ are continuous, while $\frac{d^{3} F}{d \lambda^{3}}$ discontinuously jumps. This is the original version of the GWW transition.

In the asymptotically free gauge theories which exhibit the confinement/deconfinement transition, because the coupling constant becomes smaller at higher temperature, there is an immediate identification of the weakly coupled phase of the theory with deconfinement and the strongly coupled phase of the theory with the confinement. Hence, we are tempted to see the analogy between the GWW transition in the 2D lattice theory considered above and the confinement/deconfinement transition in higher-dimensional gauge theories. Pushing this analogy, the distribution of the plaquette phases plays the role of the distribution of the Polyakov line phases. The analog of the confined phase [i.e., $\rho(\theta)=\frac{1}{2 \pi}$ for the Polyakov line phase distribution] is seen in comparing to (27) at exactly infinite coupling $\lambda=\infty$. Then, to complete the anaology for any finite coupling, $2<\lambda<\infty$, the plaquette phase distribution describes a phases of the lattice theory resembling the partially deconfined phase.

Let us make this observation more precise. Returning to the sketch of partial deconfinement in an $\mathrm{SU}(N)$ gauge theory from above, we assume a "deconfined," weakly coupled $\mathrm{SU}(M) \subset \mathrm{SU}(N)$ sector is sitting at the GWW point, while the remaining degrees of freedom are in the "confined," strongly coupled limit. Pushing the analogy to its end, the distribution (14), compared to the expectation from the generic property of partial deconfinement (27), suggests the following identification:

$$
\frac{M}{N}=\frac{2}{\lambda} \quad \text { or equivalently } \quad \lambda=\frac{2 N}{M} .
$$

Perhaps not surprisingly, the value of $\lambda$ in (30) actually corresponds to the GWW point of the $\mathrm{SU}(M)$ theory. As discussed in the previous section, the 't Hooft coupling in the truncated $\mathrm{SU}(M)$ theory is $\lambda_{M}=g^{2} M=\frac{M}{N} \cdot \lambda$. We have just seen that the GWW transition in the lattice theory at $\lambda_{M}=2$, which maps to a transition at $\lambda=\frac{2 N}{M}$ in this analogy.

Making this analogy even more precise, it follows that at the GWW transition, modulo gauge transformations, a matrix of the following form dominates the path integral:

$$
\mathcal{U}=\left(\begin{array}{cc}
\mathcal{U}_{M, \mathrm{GWW}} & \\
0 & \mathcal{U}_{N-M, \lambda=\infty}
\end{array}\right)
$$

Here, $\mathcal{U}_{M \text {.GWw }}$ is the $M \times M$ unitary matrix contributing at the GWW point, and $\mathcal{U}_{N-M, \lambda=\infty}$ is the $(N-M) \times(N-M)$ unitary matrix contributing at the strong-coupling limit. Like in the partially deconfined phase, it is convenient to think that the gauge symmetry is spontaneously broken to $\mathrm{SU}(M) \times \mathrm{SU}(N-M) \times \mathrm{U}(1)$. Further, with the above identification, one can see that at the GWW point the free energy computed from (26) on the saddle in (31) takes the same value as the free energy of the $\mathrm{SU}(M)$-truncated theory,

$$
F(\lambda)=-\frac{N^{2}}{\lambda^{2}}=-\frac{M^{2}}{4}=F_{\mathrm{GWW}}(M),
$$

where the form of the saddle point (31) allows us to interpret it simply as the sum of the contributions from the $\mathrm{SU}(M)$ and $\mathrm{SU}(N-M)$ sectors, which are $F_{\mathrm{Gww}}(M)$ and zero, respectively.

\section{B. Douglas-Kazakov transition in 2D YM on $\mathbf{S}^{\mathbf{2}}$}

Next, let us consider the large- $N$ two-dimensional $\mathrm{U}(N)$ Yang-Mills theory on 2-sphere $S^{2}$. Unlike Sec. IVA, we consider the theory at the continuum limit. The 't Hooft coupling $\lambda$ has the dimension of (mass) ${ }^{2}$, and nontrivial phase structure can be seen when the dimensionless combination $\lambda A$ is varied, where $A$ is the area of $S^{2}$ and 
$\lambda=g_{\mathrm{YM}}^{2} N$ is the 't Hooft coupling. There is a third-order phase transition similar to the GWW transition, which is called the Douglas-Kazakov (DK) transition [17]. We examine this transition following Ref. [18] and exhibit the partial-symmetry-breaking phase transition in the strong-coupling region. This theory is slightly more nontrivial compared to the previous examples, in which a simple truncation to the $\mathrm{SU}(M)$-sector worked.

The partition function is given by $Z(\lambda A, N)=$ $\int\left[d A_{\mu}\right] e^{-S}$, where the action is the usual 2D Yang-Mills on $\mathrm{S}^{2}$ with area $A$,

$$
S=-\frac{N}{4 \lambda} \int d^{2} x \sqrt{g(x)} \operatorname{Tr} F^{\mu \nu} F_{\mu \nu} .
$$

The free energy $F=-\log Z$ is given by

$$
F(\lambda A, N)=\frac{\lambda A}{24}\left(N^{2}-1\right)-\sum_{n=1}^{N} \log h_{n}\left(\frac{g_{\mathrm{YM}}^{2} A}{2}\right),
$$

where $^{10}$

$$
h_{n}\left(\frac{g_{\mathrm{YM}}^{2} A}{2}\right)=\sqrt{2 \pi}(n-1) ! \cdot\left(\frac{g_{\mathrm{YM}}^{2} A}{2}\right)^{3 / 2-n}
$$

if

$$
A<\frac{\pi^{2}}{(n-1) g_{\mathrm{YM}}^{2}},
$$

and a more complicated form at $A>\frac{\pi^{2}}{(n-1) g_{\mathrm{YM}}^{2}}$.

The form (34) is already very suggestive: $h_{1}, \ldots, h_{M}$ can be regarded as the contribution from the $\mathrm{U}(M)$-sector. As $\lambda A$ decreases from $\infty$, the DK transition takes place when the weak-coupling form (35) becomes valid for all $n=$ $1,2, \ldots, N[18]$. This is true in particular at the critical area $A_{c}\left(g_{\mathrm{YM}}^{2}, N\right)$ given by

$$
A_{c}\left(g_{\mathrm{YM}}^{2}, N\right)=\frac{\pi^{2}}{g_{\mathrm{YM}}^{2} N}=\frac{\pi^{2}}{\lambda} .
$$

As can be demonstrated easily from (34), the phase transition being probed is third order and resembles the GWW transition in the previous examples. Thus, by interpreting $(\lambda A)^{-1}$ as "temperature," we expect that the strong- and weak-coupling phases are similar to the partially deconfined and completely deconfined phases. Then, the natural expectation is that $h_{1}, h_{2}, \ldots, h_{M}$ $\left(M=\frac{\pi^{2}}{g_{\mathrm{YM}}^{A}}\right)$ are the contributions from the analog of the deconfined sector. In this context, then, $h_{M+1}, \ldots, h_{N}$ are the analog of the confined sector contributions. In order for

\footnotetext{
${ }^{10} h_{n}$ here corresponds to $h_{n-1}$ in Ref. [18].
}

this interpretation to be consistent with the partial phase transition picture, the critical area of the $\mathrm{U}(M)$ theory, $A_{\mathrm{c}}\left(g^{2}, M\right)$, has to satisfy the following consistency condition:

$$
M=\frac{\pi^{2}}{g_{\mathrm{YM}}^{2} A_{\mathrm{c}}\left(g_{\mathrm{YM}}^{2}, M\right)} .
$$

It is a trivial exercise to check that this condition is actually satisfied in the above example.

There is, perhaps, an interesting extension of the above discussion to the quantum group version of $2 \mathrm{D}$ YM. As has been noted in many places, e.g., Refs. [19,20], q-deformed $\mathrm{YM}$ on an $\mathrm{S}^{2}$ exhibits a line of third-order (DK) phase transitions much like ordinary $\mathrm{YM}$ theory for $p \geq 2$. Further, some of the intermediate phase behavior that is discussed above is hinted at explicitly in the extension of Fig. 3 in Ref. [20] to the q-deformed case, which raises an interesting question about the role of partial-symmetry-breaking phase transitions in, say, black holes and topological strings [21]. However, these are questions that we will leave for future analysis.

\section{BLACK HOLE/BLACK STRING TOPOLOGY CHANGE AND 2D SYM THEORY}

In this section, we consider $2 \mathrm{D} \mathcal{N}=(8,8) \mathrm{SU}(N) \mathrm{SYM}$ theory (i.e., with 16 supercharges). We consider the Euclidean version of the theory and put it on a torus $T^{2}=$ $S_{\beta}^{1} \times S_{L}^{1}$ where $\beta$ and $L$ are the radii of the thermal and spatial circles, respectively. For the bosonic fields, the periodic boundary condition is imposed along both circles, while for the fermionic fields, the periodic and antiperiodic boundary conditions are imposed along the spatial and temporal circles, respectively. With this boundary condition, $\beta$ is interpreted as the inverse of temperature. The action is obtained by dimensionally reducing four-dimensional (4D) $\mathcal{N}=4 \mathrm{SYM}$ to two dimensions, resulting in the action

$$
\begin{aligned}
S= & \frac{N}{\lambda_{2 \mathrm{D}}} \int_{0}^{\beta} d t \int_{0}^{L} d x \operatorname{Tr}\left(\frac{1}{4} F_{\mu \nu}^{2}+\frac{1}{2}\left(D_{\mu} X_{I}\right)^{2}-\frac{1}{4}\left[X_{I}, X_{J}\right]^{2}\right) \\
& + \text { (fermion part }),
\end{aligned}
$$

where $X_{I}(I=1,2, \ldots, 8)$ are scalar fields. There is a $\mathbb{Z}_{N_{c}}$ center symmetry, which acts on the Wilson line $W$ winding on spatial circle as $W \rightarrow e^{2 \pi i k / N} W$, where $k$ is integer. We fix $L$ and vary energy $E$ or temperature $T=1 / \beta$. As we will see, as $E$ decreases, the $\mathrm{SU}(M)$ sector goes into in the center broken phase, and $M$ gradually increases to $N$. 

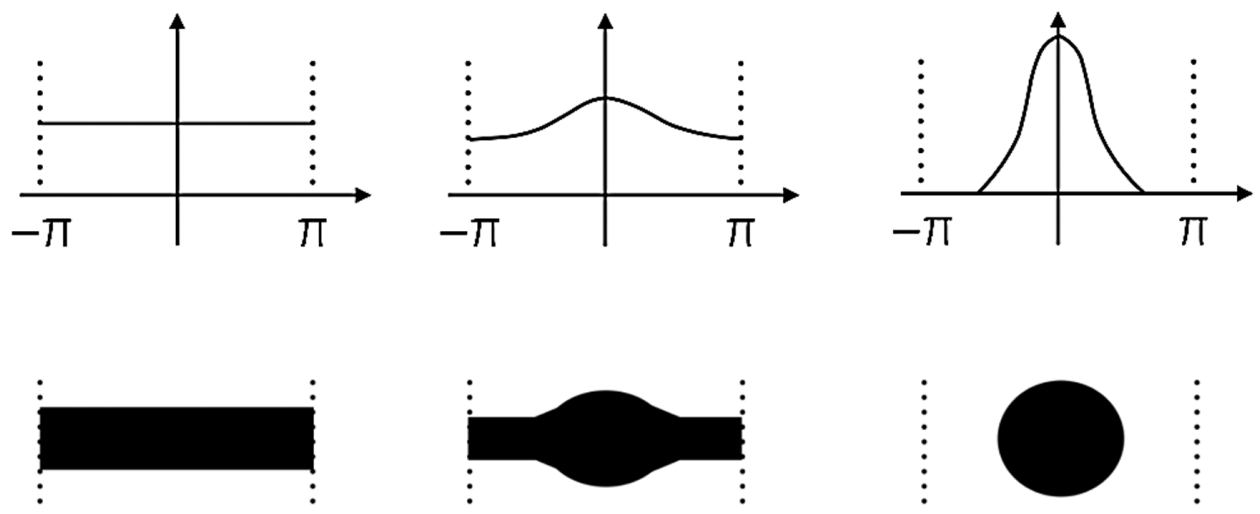

FIG. 5. The relation between the black hole/black string geometry and the distribution of the Polyakov loop [25]. (Top) the distribution of Wilson line phases, uniform (left), nonuniform but not gapped (middle), and gapped (right). (Bottom) the counterparts in gravity side. Uniform black string (left), nonuniform black string (middle), and black hole (right).

\section{A. Relation to black hole/black string topology change}

Maximally supersymmetric 2D SU(N) SYM theory captures features of the black hole/black string transition $[22,23]$ as follows [24].

First, $2 \mathrm{D} \mathcal{N}=(8,8)$ SYM theory arises in type IIB string theory as the world volume theory of $N$ D1-branes in $\mathbb{R}^{9,1}$. When the spatial dimension of the $2 \mathrm{D}$ theory is compactified, one of the nine spatial dimensions is compactified as well, and D1-branes wrap on the compactified dimension. T-dualizing along the world volume, the $N$ D1branes are mapped to $N$ D0-branes, and the circumference of the compactified circle becomes $L^{\prime} \equiv \frac{4 \pi^{2} \alpha^{\prime}}{L}$.

Staying in the T-dual picture and using the gauge/gravity correspondence, by varying $T \ll L^{-1}$ while keeping fixed the size of spatial circle $L$, the gravitational description of the D0-branes is a tiny black hole (black 0-brane). The size of the black hole is much smaller than $L^{\prime}$, and hence the solution is well approximated by that in the noncompact space. As we increase the temperature, the black hole

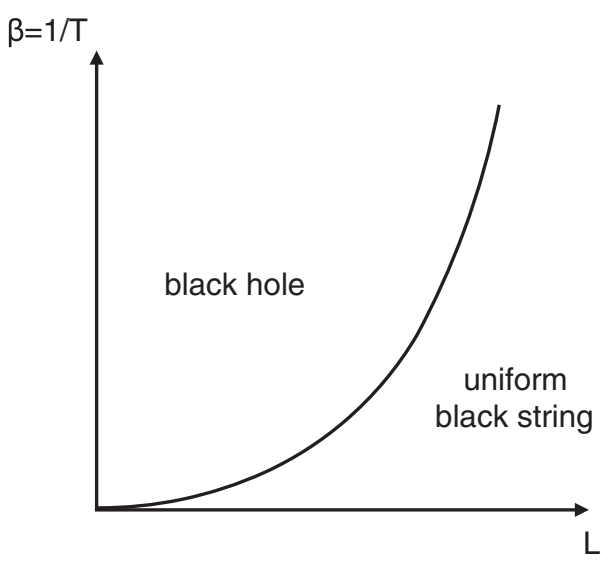

FIG. 6. A cartoon picture of phase diagram of $2 \mathrm{D}$ maximal SYM on spatial circle [25]. $L_{\mathrm{c}} \sim \beta^{1 / 3}$ at high temperature, and $L_{\mathrm{c}} \sim \beta^{1 / 2}$ at low temperature. becomes bigger and eventually wraps on the spatial circle and turns to black string. The transition temperature is higher for higher values of the (T-dual) spatial length scale $L^{\prime}$-or equivalently the transition temperature goes up as $L$ is decreased.

In terms of the SYM theory description, the locations of D0-branes are the phases of Wilson lines $W$ wrapped on the spatial $\mathrm{S}^{1}$. The distribution of Wilson line phases can be uniform, nonuniform but not gapped, or gapped; see Fig. 5 . Their gravitational descriptions are the uniform black string, nonuniform black string, and black hole, respectively. The phase diagram is shown in Fig. 6. In the following, we will detail the phase transition and find the intermediate phase.

\section{B. Small-volume, high-temperature region}

While the simulation of $2 \mathrm{D} \mathrm{SU}(N)$ SYM theory is costly for arbitrary $N$ at generic temperatures, ${ }^{11}$ at high temperature and small volume (i.e., $\beta, L \rightarrow 0$ ), the analysis simplifies as follows [24,25]. Let us start with a Euclidean path integral of the theory on $T^{2}=S_{\beta}^{1} \times S_{L}^{1}$ described above. Imposing the thermal boundary condition, the fermions are antiperiodic along the temporal circle, while bosons are periodic. Along the spatial circle, we impose periodic boundary conditions on both bosons and fermions.

At high temperature, $\beta=\frac{1}{T} \rightarrow 0$, the fermions are gapped out due to the large Kaluza-Klein mass and decouple. Integrating out the fermionic degrees of freedom and taking care of zero modes leaves behind the dimensionally reduced one-dimensional bosonic matrix model, which is a good description of the physics near $\beta=0$.

Explicitly, after the dimensional reduction, the action is given by

\footnotetext{
${ }^{11}$ See Refs. [26,27] for serious attempts and a nice summary of gauge/gravity duality in this system.
} 


$$
S_{1 \mathrm{D}}=\frac{N}{\lambda_{2 \mathrm{D}} T} \int_{0}^{L} d x \operatorname{Tr}\left(\frac{1}{2}\left(D_{x} X_{I}\right)^{2}-\frac{1}{4}\left[X_{I}, X_{J}\right]^{2}\right) .
$$

The bosonic degrees of freedom in the dimensionally reduced theory, $X_{I}(I=1,2, \ldots, 9)$, are $N \times N$ Hermitian matrices, and the gauge covariant derivative is $D_{x} X_{I}=$ $\partial_{x} X_{I}-i\left[A_{x}, X_{I}\right]$, where $A_{x}$ is the remaining component the gauge field. By rescaling $\tilde{x}=\left(\lambda_{2 \mathrm{D}} T\right)^{1 / 3} x, \tilde{L}=\left(\lambda_{2 \mathrm{D}} T\right)^{1 / 3} L$, $\tilde{D}_{x}=\left(\lambda_{2 \mathrm{D}} T\right)^{-1 / 3} D_{x}$, and $\tilde{X}_{I}=\left(\lambda_{2 \mathrm{D}} T\right)^{-1 / 3} X_{I}$, we can rewrite (40) as

$$
S_{1 \mathrm{D}}=N \int_{0}^{\tilde{L}} d \tilde{x} \operatorname{Tr}\left(\frac{1}{2}\left(\tilde{D}_{x} \tilde{X}_{I}\right)^{2}-\frac{1}{4}\left[\tilde{X}_{I}, \tilde{X}_{J}\right]^{2}\right) .
$$

In (41), when one calculates the expectation values of the form $\langle f(\tilde{X})\rangle=\frac{1}{Z} \int[d \tilde{X}] f(\tilde{X}) e^{-S}$, the only parameter is $\tilde{L}=\left(\lambda_{2 \mathrm{D}} T\right)^{1 / 3} L$, and hence large volume and high temperature are equivalent.

This high-temperature/large-volume equivalence is seen in the sketched phase diagram in Fig. 6; if one starts in the black hole phase, one can see the transition to the black string phase by either going to larger $T$ with fixed $L$ or larger $L$ with fixed $T$. The critical value of $L$ at fixed temperature scales as $L_{\mathrm{c}} \sim\left(\lambda_{2 \mathrm{D}} T\right)^{-1 / 3}$.

The computational advantage afforded by the hightemperature limit is that (41) can more easily be studied numerically using the standard Markov chain Monte Carlo (MCMC) methods (see, e.g., Refs. [28,29] for introductory articles). In the MCMC paradigm, the authors of Ref. [30] treated $\tilde{L}$ as "inverse temperature" of the dimensionally reduced theory and observed a first-order transition with hysteresis caused by the unstable saddle of the free energy [see the left panels of Figs. 2(a) and 2(b)]. Furthermore, Ref. [31] has given further evidence partial deconfinement

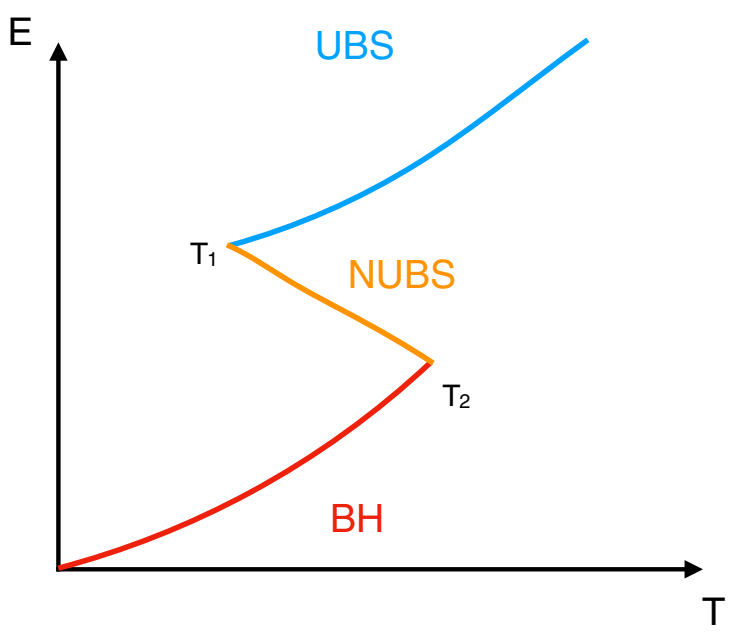

FIG. 7. A cartoon picture of the black hole/black string transition in the microcanonical ensemble, at high-T (weak coupling). Red: black hole $(\mathrm{BH})$, blue: uniform black string (UBS), and orange: nonuniform black string (NUBS). (when $\tilde{L}$ is treated as inverse temperature) of an $\mathrm{SU}(M)$ subgroup by confirming the deconfinement of the $M \times M$ submatrix in the lattice configurations in an appropriate gauge. In the 2D theory, we should see the transition of the same type when we change $T$ instead of $\tilde{L}$. Hence, the intermediate phase-nonuniform black string-should have negative specific heat. Therefore, we expect the microcanonical phase diagram for fixed small $L$ shown in Fig. 7. In the microcanonical description, the nonuniform black string phase is stable because there is only one maximum of entropy at each value of $E$. This is consistent with the classical real-time simulation [32], which observed the stable nonuniform black string phase. Note that a phase with negative specific heat corresponds to a maximum of the free energy in the canonical ensemble.

\section{Large-volume, low-temperature region}

When $L$ is sufficiently large $\left(L \gg \frac{1}{\sqrt{\lambda_{2 \mathrm{D}}}}\right)$, the transition takes place when the supergravity description of the T-dual (D0-brane) picture is a good approximation [24]. A simplified picture of the plausible microcanonical ensemble is shown in Fig. 8 with the caveat that the nonuniform string phase can be more complicated $^{12}$; there may be multiple solutions. Compared to the phase diagram in the hightemperature limit in Fig. 7, the structure of saddle points in intermediate energy scale is richer in that multiple phases can coexist. Above the critical value $E_{\mathrm{c}}$, uniform black string is entropically favored, and below $E_{\mathrm{c}}$, the black hole geometry is favored. At the intermediate energy scale, there exist other less entropically favorable saddles that are nonetheless stable in the large- $N$ limit.

If we begin in the stable uniform black string phase and gradually lower the energy, the black string remains stable until the energy reaches the Gregory-Laflamme point $E_{\mathrm{GL}}$. At $E_{\mathrm{GL}}$, the black string entropy ceases to be locally maximum, and dynamical instability to long wavelength perturbations sets in [22]. In the same manner, starting in the stable black hole phase and gradually increasing the energy, the black hole remains stable until its entropy

\footnotetext{
${ }^{12}$ There has been a long debate regarding this picture, especially regarding the final state of the Gregory-Laflamme instability [22] (see also Sec. V E). Reference [33] discussed that the black string cannot pinch off, at least in classical gravity; then, the final state is likely to be the nonuniform black string. However, Ref. [34] studied a nonuniform string solution and found that the entropy is smaller than that of uniform black string, which suggested that the nonuniform string is not the final state. Numerical simulation [35] observed the emergence of an array of black holes connected by thin black strings, but the simulation failed prior to the topology change taking place due the large gradient. References [36-39] observed that black hole and nonuniform black string meet at the topology change point, as shown in Fig. 8. By now, various solutions with the $\mathrm{S}^{1}$ compactification have been investigated, and rich phase structure has been realized; see, e.g., Refs. [40-43].
} 


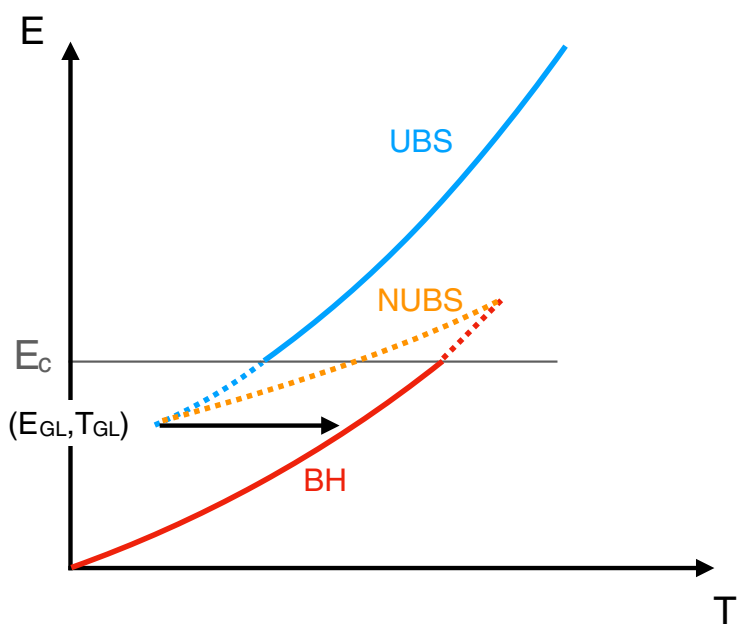

FIG. 8. Black hole/black string transition in the microcanonical ensemble. Red: black hole $(\mathrm{BH})$, blue: uniform black string (UBS), and orange: nonuniform black string (NUBS). Solid line: entropy global maximum, dotted line: local maximum (but not global maximum). The black arrow show the Gregory-Laflamme instability.

ceases to be local maximum and becomes unstable to small perturbations. When either of the initial phases reaches a point at which it becomes entropically disfavored and instability sets in, another stable branch appears-nonuniform black string - and bridges the black hole and uniform black string phases. In this sense, the microcanonical phase diagram is still continuous despite the existence of the firstorder transition at $E=E_{\mathrm{c}}$.

Different from the high-temperature limit, now the nonuniform black string phase has the positive specific heat. Thus, the nonuniform black string corresponds to a local minimum of the free energy in the canonical ensemble. In a certain temperature range, there are three stable phases, even in the canonical ensemble. ${ }^{13}$

\section{Nonuniform black string as partial-symmetry-breaking phase}

The intermediate, nonuniform black string phase can be regarded as the partial-symmetry-breaking phase in which the $\mathrm{SU}(N)$ gauge symmetry is spontaneously broken to $\mathrm{SU}(M) \times \mathrm{SU}(N-M)$. As demonstrated in Refs. [30,31] for the high-temperature limit, the distribution of the phases of the Wilson loop wrapped on the spatial circle gives the gauge-invariant order parameter: the constant offset agrees with $\frac{M}{N}$. An important point to note in this phase is that the $\mathbb{Z}_{N}$ center symmetry is completely broken. Effectively, the $\mathbb{Z}_{N-M}$ center symmetry is realized in the confined sector, but it is not a subgroup of the $\mathbb{Z}_{N}$ symmetry.

\footnotetext{
${ }^{13}$ Strictly speaking, two of the phases are metastable. However, in the large- $N$ limit, the tunneling is parametrically suppressed as the probability of such an event is $\mathcal{O}\left(e^{-N^{2}}\right)$.
}

One natural question is as follows. We claimed that the specific heat is negative in the nonuniform black string phase at high temperature and small volume, and it exists stably in a microcanonical ensemble. Why is it possible? Why do we not have the coexistence of two phases separated in volume? To understand it, first recall the argument in Sec. II B 1: at small volume, whether the thermodynamic instability sets in depends on the detail of the dynamics. The case under consideration is the center symmetry breaking phase transition, which is essentially the onset of the decoupling of the Kaluza-Klein (KK) mode. After the KK modes decouple, the system has to be uniform along the spatial circle and hence cannot occupy a small fraction of the physical space.

\section{E. Stringy effects eliminate the Gregory-Laflamme instability?}

It is interesting to see how stringy corrections can affect the Gregory-Laflamme instability of the uniform black string [22]. The Gregory-Laflamme instability means the instability of the uniform black string against a small perturbation, which is observed in classical gravity. It is believed that the black string eventually pinches off and become black hole; although a more complete understanding of quantum gravity is needed to resolve the singularity associated with the topology change.

In terms of dual gauge theory, it is the instability expressed by the black arrow in Fig. 8. Note that this is a first-order transition in the microcanonical ensemble. At high temperature and small volume, however, there seems to be no first-order transition in the microcanonical ensemble (Fig. 7). Maybe a small jump exists near the border between the uniform black string and nonuniform black string, or the one between the nonuniform black string and black hole, but at least the transition from uniform black string to black hole is impossible. It suggests that a large stringy correction eventually eliminates the Gregory-Laflamme instability.

Note that a similar transition in the canonical ensemble survives. Namely, if the temperature is gradually lowered, a jump from black string to black hole at the same temperature but smaller energy takes place.

\section{YANG-MILLS WITH ADJOINT FERMIONS ON $\mathbb{R}^{3} \times \mathrm{S}^{1}$}

Let us consider another example where it is very easy to see the gauge symmetry breaking: $\mathrm{SU}\left(N_{c}\right)$ Yang-Mills theory with $N_{f}$ Majorana adjoint fermions of mass $m$ on $\mathbb{R}^{3} \times S^{1}$. Similarly to some other examples above, this theory has been studied extensively, but a simple and direct connection of the observed phase behavior to partial symmetry breaking has not been understood.

For the example below, we will denote the radius of the $S^{1}$ by $L$ and consider cases where $N_{f} \geq 2$. We impose 
periodic boundary conditions both for the gauge field and adjoint fermions around the $\mathrm{S}^{1}$. When $L$ is small, the dimensionally reduced three-dimensional picture is good, and the gauge field along the $\mathrm{S}^{1}$ can be regarded as an adjoint Higgs field. At $L \ll m^{-1}$, the usual spontaneous symmetry breaking takes place, as we will see below.

Staying in the regime of fixed small $L$ and large $m$, the phase diagram of this theory can be analyzed (to some extent) by using the one-loop effective action [44]

$$
\begin{aligned}
V[\Omega] & =\frac{2}{\pi^{2} L^{4}} \sum_{n=1}^{\infty}\left\{-1+\frac{n_{f}}{2}(n L m)^{2} K_{2}(n L m)\right\} \frac{\left|\operatorname{Tr} \Omega^{n}\right|^{2}}{n^{4}} \\
& \equiv \sum_{n=1}^{\infty} f_{n}(L m)\left|\operatorname{Tr} \Omega^{n}\right|^{2}
\end{aligned}
$$

where $\Omega=\operatorname{diag}\left(e^{i \theta_{1}}, \ldots, e^{i \theta_{N}}\right)$ is the Wilson line wrapping on the spatial circle and $K_{2}(z)$ is the modified Bessel function of the second kind. The free energy is obtained by minimizing the effective action with respect to $\Omega$.

Let $z=z_{0}$ be the solution of $-1+\frac{n_{f}}{2}(z)^{2} K_{2}(z)=0$. Since $z^{2} K_{2}(z)$ is monotonically decreasing in the region $z>0$, at $\frac{z_{0}}{m}<L$, all $f_{j}(L m)$ 's are negative. Thus, the minimum of the effective action is obtained by setting $\left|\operatorname{Tr} \Omega^{n}\right|=1$ for any $n$, or equivalently $\theta_{1}=\theta_{2}=\cdots \theta_{N}$. This solution to the saddle point equation is the $\mathbb{Z}_{1}$-centersymmetry phase (i.e., the center symmetry is completely broken), and correspondingly the solution preserves the full $\mathrm{SU}(N)$ gauge symmetry.

If we start to dial the size of the $\mathrm{S}^{1}$ such that $L$ becomes smaller, the coefficient $f_{n}(L m)$ turns positive at $L<\frac{z_{0}}{n m}$, and the center symmetry is restored as [44]

$$
\mathbb{Z}_{1} \rightarrow \mathbb{Z}_{2} \rightarrow \mathbb{Z}_{3} \rightarrow \cdots
$$

The center symmetry restoration coincides with the gauge symmetry breaking pattern

$$
\begin{aligned}
\mathrm{SU}(N) & \rightarrow[\mathrm{SU}(N / 2)]^{2} \times \mathrm{U}(1) \rightarrow[\mathrm{SU}(N / 3)]^{3} \times[\mathrm{U}(1)]^{2} \\
& \rightarrow \cdots .
\end{aligned}
$$

Apparently, an intermediate phase must exist between $\mathbb{Z}_{k^{-}}$ and $\mathbb{Z}_{k+1}$-center-symmetric phases.

In order to understand the appearance and meaning of the intermediate phase, let us start by considering the simplest case $\mathbb{Z}_{1} \rightarrow \mathbb{Z}_{2}$. In terms of gauge symmetry breaking, we want to study the phase connecting $\mathrm{SU}(N)$ and $\mathrm{SU}(N / 2) \times \mathrm{SU}(N / 2)$, which naively will interpolate $\mathrm{SU}(N-M) \times \mathrm{SU}(M)$ from $M=0$ to $M=N / 2$.

We start with a simple ansatz of a block diagonal form for the holonomy matrix in the intermediate phase,

$$
\Omega=\left(\begin{array}{cc}
\mathbb{1}_{\mathrm{N}-\mathrm{M}} & 0 \\
0 & -\mathbb{1}_{\mathrm{M}}
\end{array}\right),
$$

where $\mathbb{1}_{d}$ is the $d$-dimensional unit matrix. It is straightforward, then to show that on this ansatz $\operatorname{Tr} \Omega^{n}=N$ for even $n$ and $\operatorname{Tr} \Omega^{n}=N-2 M$ for odd $n$. Evaluating the effective action on the ansatz gives

$$
\begin{aligned}
V[\Omega] & =\sum_{n: \text { even }} f_{n}(L m) N^{2}+\sum_{n: \text { odd }} f_{n}(L m)(N-2 M)^{2} \\
& =\left(1-\frac{2 M}{N}\right)^{2} V_{1}+\left(1-\left(1-\frac{2 M}{N}\right)^{2}\right) V_{2},
\end{aligned}
$$

where $V_{1}$ and $V_{2}$ are the free energies of the $\mathbb{Z}_{1}$ and $\mathbb{Z}_{2}$ configurations, respectively. When $V_{1}=V_{2}$ (namely, $\sum_{n \text { :odd }} f_{n}(L m)=0$ ), the free energy does not depend on $M$, and hence the first-order transition without hysteresis appears.

To understand other intermediate phases that appear in the sequence $\mathbb{Z}_{k} \rightarrow \mathbb{Z}_{k+1}$, let us consider $\mathbb{Z}_{2} \rightarrow \mathbb{Z}_{3}$, which is still relatively simple to discuss and contains the salient features to understand generic $k$. As an intermediate phase, there is an $\mathrm{SU}(M) \times \mathrm{SU}(M) \times \mathrm{SU}(N-2 M) \times \mathrm{U}(1)^{2}$ phase. The holonomy matrix should then take the form

$$
\Omega=\left(\begin{array}{ccc}
\phi \mathbb{1}_{M} & 0 & 0 \\
0 & \phi^{*} \mathbb{1}_{M} & 0 \\
0 & 0 & \mathbb{1}_{N-2 M}
\end{array}\right) .
$$

Note that $\phi=i$ when $M=N / 2$ and $\phi=e^{2 \pi i / 3}$ when $M=N / 3$. At each $M, \phi$ can be evaluated by extremizing the effective action within this ansatz. It is straightforward, though tedious, to generalize the argument above to generic $\mathbb{Z}_{k} \rightarrow \mathbb{Z}_{k+1}$ intermediate phases. Note that Ref. [45] studied several finite- $N$ theories and found analogous phases which are stable.

Note that the center symmetry changes abruptly. It is completely broken in the intermediate phase connecting $\mathbb{Z}_{k}$ and $\mathbb{Z}_{k+1}$ phases, while the gauge symmetry changes only gradually. Similarly to the case of the confinement/deconfinement transition, we can understand the system much better by focusing on the gauge symmetry, rather than the center symmetry.

\section{CONCLUSIONS}

In this paper, we have examined a variety of theories with large rank symmetry groups and observed that partialsymmetry-breaking phase transitions appear to be generic large- $N$ phenomena leading to interesting phase coexistence behavior. In each case, the partial-symmetry-breaking phase appears as an intermediate phase connecting two (meta)stable phases that generically realize the full rank symmetry group. While the examples we have discussed are gauge theories, the underlying mechanism discussed in $\mathrm{Sec}$. II does not fundamentally require a gauge symmetry at all. This leads somewhat naturally to our speculation that such partial-symmetry-breaking phase transitions could 
admit a broad universality class. After all, the demonstration of these novel transitions through matrix models certainly suggests that theories that have limiting descriptions in terms of random matrices could exhibit partial symmetry in intermediate phases as well. However, it is unclear how to construct a generic proof of universality, and so it would be worthwhile to explore possible examples that lie outside of the scope of gauge theories.

While we have focused exclusively on examples in gauge theories here (apart from the toy model of ant trails in Appendix), there are a number of ways in which partialsymmetry-breaking phase transitions could appear in other areas of physics. Indeed, there seem to be natural analogs for these transitions observed in the theories we have studied above in phenomena in statistical physics models such as quantum rotors, Dimer models, and Bose-Einstein condensates that could be well-described by the same language. While it is not immediately clear what, if any, immediate utility this reinterpretation could have, it may be worth trying to understand the connections between confinement/deconfinement and phase transitions in condensed matter that could afford a more unified picture of the fundamental physics governing them. ${ }^{14}$

Returning to gauge theoretic examples, one example where one might expect to possibly see partial-symmetrybreaking phase transitions would be in a particular deformation of the index (or rather the supersymmetric partition function on $S^{1} \times S^{d-1}$ ) of superconformal field theories. It was noted in Ref. [46] that in analytically continuing fugacities, $x$, in the superconformal index of four-dimensional $\mathcal{N}=4 \mathrm{SU}(N) \mathrm{SYM}$ theory, one could observe a confinement/deconfinement transition by taking $x$ to be a complex number. It was previously argued that, unlike the partition function, supersymmetric indices are invariant under changes in continuous parameters, and so phase transitions would not be captured by any index computation [47]. It would be interesting to see if this deformed index does see any partial-symmetry-breaking phase transitions between the confined and deconfined phases; if such partial-symmetry-breaking phase transitions are visible in the index with complex fugacities and given the vast literature on supersymmetric and superconformal indices, it would also be interesting to see any broader implications.

It is also interesting to note that we have seen in Sec. III A that partial deconfinement can happen in QCD-like theories. A consequence of the partial-symmetry-breaking phase transition is that a simultaneous breaking and restoration of gauge symmetry is involved, which may give a precise definition of confinement/deconfinement transition based on the change of symmetry [4]. (Note that

\footnotetext{
${ }^{14}$ After the first version of this paper appeared in the arXiv, Ref. [10] pointed out a concrete connection between the BoseEinstein condensation and partial-symmetry-breaking phase transitions.
}

it is widely believed that there is no symmetry characterizing deconfinement because center symmetry is explicitly broken by the existence of quarks in the fundamental representation.) It is important to understand whether the phenomenon we have discovered survives at $N_{c}=3$ and infinite volume and whether there are consequences in observable quantities.

\section{ACKNOWLEDGMENTS}

We thank Tom Cohen, Oscar Dias, Nick Evans, Pau Figueras, Antal Jevicki, Tatsuhiro Misumi, Andy O'Bannon, Cheng Peng, Masaki Tezuka, Hiromasa Watanabe, Nico Wintergerst, Toby Wiseman, and Naoki Yamamoto for stimulating discussions and comments. The work of M. H. was partially supported by the STFC Ernest Rutherford Grant No. ST/R003599/1 and JSPS KAKENHI Grant No. 17K1428. The work of B. R. was partially supported through the STFC Consolidated Grant No. ST/L000296/1 and by the KU Leuven C1 Grant No. ZKD1118 C16/16/005.

\section{APPENDIX: ANT TRAIL FORMATION}

Departing entirely from high energy physics, let us consider a somewhat funny example from entomology: the formation of an ant trail [48]. This cute, relatively simple, phenomenological model has a phase behavior that strikingly resembles the brane model [3].

To summarize the ant model in Ref. [48], consider a colony of $N$ indistinguishable ants such that there exists an $\mathrm{S}_{N}$ permutation symmetry exchanging any individual member of the colony. The number of ants forming a trail as a function of time is denoted $M(t)$ and depends on a number of environmental factors - e.g. local topography, weather, and so on-such that the time dependence of $M$ is modeled as

$$
\frac{d M}{d t}=(\alpha+p M)(N-M)-\frac{s M}{s+M} .
$$

The parameters in Eq. (A1) encode the system dynamics as $\alpha$ specifying the probability that each ant finds the food source accidentally, $s$ describing the rate that ants leave the trail, and $p$ giving the strength of the pheromone-mediated nonlocal twobody interaction between each ant. To give a sense of the environmental dependence in the model, consider a day with particularly dry air conditions; with the lack of ambient moisture in the air, the pheromone evaporates faster, and hence $p$ becomes smaller. The size of the stationary ant trail can be obtained by solving the equation $\frac{d M}{d t}=0$.

While it is true that $M$ can change continuously, some care is needed with regard to the distinction between canonical and microcanonical ensembles. We follow the treatment in Ref. [3], in order to make the application to physics problems more salient. That is, there are analogous 

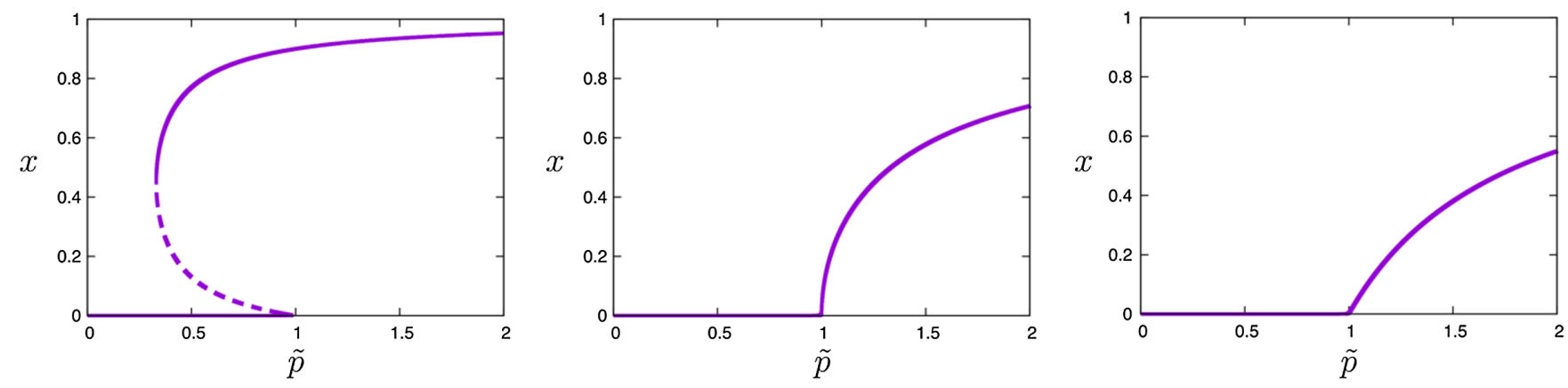

FIG. 9. $x \equiv \frac{M}{N}$ vs $\tilde{p} \equiv N p$ in the ant trail model (A1). $N \alpha=1, \tilde{s} \equiv \frac{s}{N}=0.1$ (left), 1.0 (center), and 5.0 (right), $N=10^{5}$. Similar figures have been used in Ref. [3].

features shared between the stationary trail equation and the saddle point equation for other systems we discuss later.

An interesting "large- $N$ " limit of the ant model is taken where $\alpha \sim N^{-1}, p \sim N^{-1}$, and $s \sim N^{1}$ [3]. In Fig. 9, we show the plot of $x \equiv \frac{M}{N}$ versus $\tilde{p} \equiv N p$ for $N \alpha=1$, several $\mathcal{O}(1)$ values of $\tilde{s} \equiv \frac{s}{N}$, and $N=10^{5}$. As we will explain later, $\tilde{p}$ is analogous to temperature in the brane model [3]. The number of the ants $M$ corresponds to the number of $\mathrm{D}$ branes in the bound state.

In the three examples of varying $\tilde{s}$ increasing from left to right in Fig. 9, we see the stable - or possibly metastable saddles (solid lines) and unstable saddles (dashed lines) displaying interesting phase behavior. Whether the saddle is stable or unstable can be seen from the sign of $\frac{d M}{d t}$ around the saddle. Note that here we vary $\tilde{p}$ and study the response in $M$, which amounts to being in the canonical ensemble. Starting from the leftmost panel in Fig. 9, we have the following:

(i) For $\tilde{s}<1$ in the left panel of Fig. 9, if under small perturbations to $M$ away from the saddle the trail accumulates new ants or disintegrates, the saddle is unstable (dashed line). This unstable saddle separates two (meta)stable saddles and causes the firstorder phase transition.

In order for the tunneling between two stable saddles to happen, it is necessary to go beyond the unstable saddle. It requires $\mathcal{O}(N)$ change of the number of the ants, and hence as $N$ grows it becomes harder and harder to go across this unstable phase. Therefore, there is no tunneling in the large- $N$ limit. (ii) When $\tilde{s}>1$ in the right panel of Fig. 9, under small perturbations to $M$, the saddle is stable at all $\tilde{p}$.

(iii) At $\tilde{s}=1$ in the center panel of Fig. 9, we see the boundary between two cases where the curve $x=$ $x(\tilde{p})$ has infinite slope at $\tilde{p}=1$ at large $N$.

It is easy to find the analogy to the system of D-branes [3]. At higher temperature, each open string mode can be excited more, and hence the attraction becomes stronger; the analog in ant trail formation is that the nonlocal coupling (pheromone) attracting ants to the trail becomes stronger. The suppression of $M$ due to the Boltzmann factor balancing the entropic force is played by the "laziness" term in the ant model, which describes the rate of the ants leaving the trail. Just as was seen in the brane model where bound states formed at the point where the entropic forces and Boltzmann suppression were balanced, a stationary phase exists for the ant trail where the outflow due to the laziness effect is balanced against the inflow due to the strength of pheromone.

The internal space consisting of the ants splits into two simultaneously realized phases: inside and outside the trail. Hence, two phases can coexist in the internal space. The permutation symmetry of the ants breaks as $S_{N} \rightarrow S_{M} \times$ $S_{N-M}$, and hence partial symmetry breaking can occur. The analog of temperature is the pheromone parameter $\tilde{p}$. As we can see from Fig. 9, $\tilde{p}$ can change nontrivially as a function of $x$. Finally, in the left panel of Fig. 9, $\mathcal{O}(N)$ perturbation to the number of ants on the trail is needed in order for the "tunneling" across the unstable trail to happen, and hence the metastable phase can be completely stabilized in the thermodynamic limit (many-ant limit).
[1] M. Hanada and J. Maltz, A proposal of the gauge theory description of the small Schwarzschild black hole in $\mathrm{AdS}_{5} \times \mathrm{S}^{5}$, J. High Energy Phys. 02 (2017) 012.

[2] D. Berenstein, Submatrix deconfinement and small black holes in AdS, J. High Energy Phys. 09 (2018) 054.
[3] M. Hanada, G. Ishiki, and H. Watanabe, Partial deconfinement, J. High Energy Phys. 03 (2019) 145.

[4] M. Hanada, A. Jevicki, C. Peng, and N. Wintergerst, Anatomy of deconfinement, J. High Energy Phys. 12 (2019) 167. 
[5] J. Dai, R. G. Leigh, and J. Polchinski, New connections between string theories, Mod. Phys. Lett. A 04, 2073 (1989).

[6] E. Witten, Bound states of strings and p-branes, Nucl. Phys. B460, 335 (1996).

[7] B. Sundborg, The Hagedorn transition, deconfinement and $N=4$ SYM theory, Nucl. Phys. B573, 349 (2000).

[8] O. Aharony, J. Marsano, S. Minwalla, K. Papadodimas, and M. Van Raamsdonk, The Hagedorn-deconfinement phase transition in weakly coupled large $\mathrm{N}$ gauge theories, Adv. Theor. Math. Phys. 8, 603 (2004); 8, 161 (2003).

[9] E. Berkowitz, M. Hanada, and J. Maltz, Chaos in matrix models and black hole evaporation, Phys. Rev. D 94, 126009 (2016).

[10] M. Hanada, H. Shimada, and N. Wintergerst, Color confinement and Bose-Einstein condensation, arXiv:2001 .10459 .

[11] A. Einstein, Quantentheorie des einatomigen idealen gases. Zweite Abhandlung, in Albert Einstein: Akademie Vorträge (John Wiley \& Sons, Ltd, 1924), pp. 245-257, ISBN 9783527608959, https://doi.org/10.1002/3527608958 .ch28.

[12] H. J. Schnitzer, Confinement/deconfinement transition of large $\mathrm{N}$ gauge theories with $\mathrm{N}(\mathrm{f})$ fundamentals: $\mathrm{N}(\mathrm{f}) / \mathrm{N}$ finite, Nucl. Phys. B695, 267 (2004).

[13] T. J. Hollowood and J. C. Myers, Deconfinement transitions of large N QCD with chemical potential at weak and strong coupling, J. High Energy Phys. 10 (2012) 067.

[14] D. J. Gross and E. Witten, Possible third order phase transition in the large $\mathrm{N}$ lattice gauge theory, Phys. Rev. D 21, 446 (1980).

[15] S. R. Wadia, A study of $U(N)$ lattice gauge theory in 2-dimensions, arXiv:1212.2906.

[16] C. Vafa and E. Witten, Restrictions on symmetry breaking in vector-like gauge theories, Nucl. Phys. B234, 173 (1984).

[17] M. R. Douglas and V. A. Kazakov, Large N phase transition in continuum QCD in two-dimensions, Phys. Lett. B 319, 219 (1993).

[18] D. J. Gross and A. Matytsin, Instanton induced large N phase transitions in two-dimensional and four-dimensional QCD, Nucl. Phys. B429, 50 (1994).

[19] X. Arsiwalla, R. Boels, M. Marino, and A. Sinkovics, Phase transitions in q-deformed 2-D Yang-Mills theory and topological strings, Phys. Rev. D 73, 026005 (2006).

[20] D. Jafferis and J. Marsano, A DK phase transition in q-deformed Yang-Mills on $\mathrm{S}^{* * 2}$ and topological strings, arXiv:hep-th/0509004.

[21] M. Aganagic, H. Ooguri, N. Saulina, and C. Vafa, Black holes, q-deformed 2d Yang-Mills, and non-perturbative topological strings, Nucl. Phys. B715, 304 (2005).

[22] R. Gregory and R. Laflamme, Black Strings and p-Branes are Unstable, Phys. Rev. Lett. 70, 2837 (1993).

[23] B. Kol, Topology change in general relativity, and the black hole black string transition, J. High Energy Phys. 10 (2005) 049.

[24] O. Aharony, J. Marsano, S. Minwalla, and T. Wiseman, Black hole-black string phase transitions in thermal $1+1$ dimensional supersymmetric Yang-Mills theory on a circle, Classical Quantum Gravity 21, 5169 (2004).

[25] O. Aharony, J. Marsano, S. Minwalla, K. Papadodimas, M. Van Raamsdonk, and T. Wiseman, The Phase structure of low dimensional large $\mathrm{N}$ gauge theories on Tori, J. High Energy Phys. 01 (2006) 140.

[26] S. Catterall, A. Joseph, and T. Wiseman, Thermal phases of D1-branes on a circle from lattice super Yang-Mills, J. High Energy Phys. 12 (2010) 022.

[27] S. Catterall, R. G. Jha, D. Schaich, and T. Wiseman, Testing holography using lattice super-Yang-Mills theory on a 2-torus, Phys. Rev. D 97, 086020 (2018).

[28] M. Hanada, Markov chain Monte Carlo for dummies, arXiv:1808.08490.

[29] T. DeGrand, Lattice methods for students at a formal TASI, in Theoretical Advanced Study Institute in Elementary Particle Physics: The Many Dimensions of Quantum Field Theory (TASI 2019) Boulder, CO, USA, 2019 (2019), https://physicslearning.colorado.edu/tasi/ tasi_2019/tasi_2019.html.

[30] G. Bergner, N. Bodendorfer, M. Hanada, E. Rinaldi, A. Schafer, and P. Vranas, Thermal phase transition in Yang-Mills matrix mod el, J. High Energy Phys. 01 (2020) 053.

[31] H. Watanabe, G. Bergner, N. Bodendorfer, S. Shiba Funai, M. Hanada, E. Rinaldi, A. Schaefer, and P. Vranas, Partial deconfinement at strong coupling on the lattice, arXiv: 2005.04103.

[32] M. Hanada and P. Romatschke, Real time quantum gravity dynamics from classical statistical Yang-Mills simulations, J. High Energy Phys. 01 (2019) 201.

[33] G. T. Horowitz and K. Maeda, Fate of the Black String Instability, Phys. Rev. Lett. 87, 131301 (2001).

[34] T. Wiseman, Static axisymmetric vacuum solutions and nonuniform black strings, Classical Quantum Gravity 20, 1137 (2003).

[35] M. W. Choptuik, L. Lehner, I. Olabarrieta, R. Petryk, F. Pretorius, and H. Villegas, Towards the final fate of an unstable black string, Phys. Rev. D 68, 044001 (2003).

[36] H. Kudoh and T. Wiseman, Connecting Black Holes and Black Strings, Phys. Rev. Lett. 94, 161102 (2005).

[37] B. Kleihaus, J. Kunz, and E. Radu, New nonuniform black string solutions, J. High Energy Phys. 06 (2006) 016.

[38] M. Headrick, S. Kitchen, and T. Wiseman, A new approach to static numerical relativity, and its application to KaluzaKlein black holes, Classical Quantum Gravity 27, 035002 (2010).

[39] M. Kalisch, S. Mockel, and M. Ammon, Critical behavior of the black hole/black string transition, J. High Energy Phys. 08 (2017) 049.

[40] O. J. C. Dias, T. Harmark, R. C. Myers, and N. A. Obers, Multi-black hole configurations on the cylinder, Phys. Rev. D 76, 104025 (2007).

[41] P. Figueras, K. Murata, and H. S. Reall, Stable non-uniform black strings below the critical dimension, J. High Energy Phys. 11 (2012) 071.

[42] R. Emparan, P. Figueras, and M. Martinez, Bumpy black holes, J. High Energy Phys. 12 (2014) 072. 
[43] O. J. C. Dias, J.E. Santos, and B. Way, Localised and nonuniform thermal states of super-Yang-Mills on a circle, J. High Energy Phys. 06 (2017) 029.

[44] M. Unsal and L. G. Yaffe, Large-N volume independence in conformal and confining gauge theories, J. High Energy Phys. 08 (2010) 030.

[45] J. C. Myers and M. C. Ogilvie, Phase diagrams of SU(N) gauge theories with fermions in various representations, J. High Energy Phys. 07 (2009) 095.
[46] S. Choi, J. Kim, S. Kim, and J. Nahmgoong, Comments on deconfinement in AdS/CFT, arXiv:1811.08646.

[47] J. Kinney, J. M. Maldacena, S. Minwalla, and S. Raju, An Index for 4 dimensional super conformal theories, Commun. Math. Phys. 275, 209 (2007).

[48] M. Beekman, D. J. T. Sumpter, and F. L. W. Ratnieks, Phase transition between disordered and ordered foraging in pharaoh's ants, Proc. Natl. Acad. Sci. U.S.A. 98, 9703 (2001). 